\title{
A TWO-Factor MOdel for StOchastic MORTALITY WITH PARAMETER UnCERTAINTY: THEORY AND CALIBRATION
}

\author{
Andrew J. G. Cairns \\ David Blake \\ Kevin Dowd
}

\begin{abstract}
In this article, we consider the evolution of the post-age- 60 mortality curve in the United Kingdom and its impact on the pricing of the risk associated with aggregate mortality improvements over time: so-called longevity risk. We introduce a two-factor stochastic model for the development of this curve through time. The first factor affects mortality-rate dynamics at all ages in the same way, whereas the second factor affects mortality-rate dynamics at higher ages much more than at lower ages. The article then examines the pricing of longevity bonds with different terms to maturity referenced to different cohorts. We find that longevity risk over relatively short time horizons is very low, but at horizons in excess of ten years it begins to pick up very rapidly.

A key component of the article is the proposal and development of a method for calculating the market risk-adjusted price of a longevity bond. The proposed adjustment includes not just an allowance for the underlying stochastic mortality, but also makes an allowance for parameter risk. We utilize the pricing information contained in the November 2004 European Investment Bank longevity bond to make inferences about the likely market prices of the risks in the model. Based on these, we investigate how future issues might be priced to ensure an absence of arbitrage between bonds with different characteristics.
\end{abstract}

\footnotetext{
Andrew J. G. Cairns is at Maxwell Institute for Mathematical Sciences, Edinburgh, and Actuarial Mathematics and Statistics, Heriot-Watt University, Edinburgh, EH14 4AS, United Kingdom. David Blake is at Pensions Institute, Cass Business School, City University, 106 Bunhill Row, London, EC1Y 8TZ, United Kingdom. Kevin Dowd is at Centre for Risk \& Insurance Studies, Nottingham University Business School, Jubilee Campus, Nottingham, NG8 1BB, United Kingdom. The author can be contacted via e-mail: A.Cairns@ma.hw.ac.uk. The authors would like to thank two anonymous referees for their very helpful observations and comments. AC wishes to thank the Isaac Newton Institute in Cambridge, where he was a visitor during the preparation of this paper. This research was conducted under research grants RES-000-27-0014 and RES-000-23-1036 from the UK Economic and Social Research Council.
} 


\section{INTRODUCTION}

Recently, it has become clear that mortality is a stochastic process: longevity has not only been improving, but it has been improving, to some extent, in an unpredictable way. These unanticipated improvements have proved to be of greatest significance at higher ages, and have caused life offices (and pension plan sponsors in the case where the plan provides the pension) to incur losses on their life annuity business. The problem is that pensioners are living much longer than was anticipated, say, twenty years ago. As a result, life offices are paying out for much longer than was anticipated, and their profit margins are being eroded in the process. The insurance industry is therefore bearing the costs of unexpectedly greater longevity. Looking forward, possible changes in lifestyle, medical advances, and new discoveries in genetics are likely to make future improvements to life expectancy highly unpredictable as well. This, in turn, will lead to smaller books of life annuity business, smaller profit margins, or both.

There are a number of possible types of systematic, mortality-related risks that annuity providers and life insurers are exposed to. For the sake of clarity, in this article we will use the following conventions.

- The term mortality risk should be taken to encompass all forms of uncertainty in future mortality rates, including increases and decreases in mortality rates.

- Longevity risk should be interpreted as uncertainty in the long-term trend in mortality rates and its impact on the long-term probability of survival of an individual. Longevity risk is normally taken to mean the risk that survival rates are higher than anticipated, although we strictly take it to mean uncertainty in either direction.

- Short-term, catastrophic mortality risk should be interpreted as the risk that, over short periods of time, mortality rates are much higher (or lower) than would normally be experienced. Examples of such "catastrophes" include the influenza pandemic in 1918 and the tsunami in December 2004. Once the catastrophe has past, we expect mortality rates to revert to their previous levels and to continue along previous trends. ${ }^{1}$

The idea of using the capital markets to securitize and trade specific insurance risks is relatively new, and picked up momentum in the 1990s with a number of securitizations of non-life insurance risks (see, for example, Lane, 2000). December 2003 saw the issue by Swiss Re of the first bond to link payments to mortality risk: specifically short-term, catastrophic mortality risk. A related capital market innovation, the longevity bond, provides life offices and pension plans with an instrument to hedge the much-longerterm longevity risks that they face. The idea for longevity bonds was first published in the Journal of Risk and Insurance in 2001. ${ }^{2}$ Longevity bonds are annuity bonds whose coupons are not fixed over time, but fall in line with a given survivor index. ${ }^{3}$ For

\footnotetext{
${ }^{1}$ Note that long-term trends in mortality might, however, be affected by certain types of catastrophe. For example, survivors of a severe outbreak of influenza might be weakened in some way and more prone in the future to heart disease or cancer. In this sense, catastrophic mortality events might be correlated with long-term trends.

${ }^{2}$ Blake and Burrows (2001). See also Cox, Fairchild, and Pedersen (2000).

${ }^{3}$ For this reason they are also known as survivor bonds (e.g., Blake, and Burrows, 2001).
} 
example, the survivor index might be based on the population of 65-year olds alive on the issue date of the bond. Each year the coupon payments received by the life office or pension plan decrease by the percentage of the population who have died that year. If, after the first year, $1.5 \%$ of the population of what are now 66-year olds have died, then the coupon payable at the end of that first year will fall to $98.5 \%$ of the nominal coupon rate. But this is exactly what the life office or pension plan wants, since only $98.5 \%$ of their own 66-year-old annuitants (assuming these are representative of reference population) will be alive after one year, so they do not have to pay out so much.

In November 2004, BNP Paribas (in its role as structurer and manager) announced that the European Investment Bank (EIB) would issue a longevity bond. The bond had an initial market value of about $£ 540 \mathrm{~m}$ and a maturity of twenty-five years. Its coupon payments were to be linked to a survivor index based on the realized mortality experience of a cohort of males from England \& Wales aged 65 in 2003 as published by the UK Office for National Statistics (ONS). The intended main investors were UK pension funds and life offices. ${ }^{4}$ Although this issue was ultimately unsuccessful, there are important issues to be learned about how to price such contracts (an issue which we discuss at length in this article) and about design issues (which are discussed elsewhere: see, for example, Blake, Cairns, and Dowd, 2006).

The basic cashflows under the EIB/BNP longevity bond, ignoring credit risk, are described in Appendix A. Our article focuses on the mathematical modelling that underpins the pricing of mortality-linked securities. For a full discussion of the EIB/BNP bond as well as other types of mortality-linked security, the reader is referred to Cowley and Cummins (2005), Cairns, et. al. (2005), and Blake, Cairns, and Dowd (2006).

A variety of approaches have been proposed for modelling the randomness in aggregate mortality rates over time. A key earlier work is that of Lee and Carter (1992). Their work focuses on the practical application of stochastic mortality and its statistical analysis. Aggregate mortality rates are, at best, measured annually and for this reason Lee and Carter (1992) and later authors who adopted a similar approach (see, for example, Brouhns, Denuit, and Vermunt, 2002; Renshaw and Haberman, 2003; Currie, Durban, and Eilers, 2004) worked in discrete time. Models following the approach of Lee and Carter typically adapt discrete-time time series models to capture the random element in the stochastic development of mortality rates. Other authors have developed models in a continuous-time framework (see, for example, Milevsky and Promislow, 2001; Dahl, 2004; Dahl and Møller, 2005; Miltersen and Persson, 2005; Biffis, 2005; Schrager, 2006). For further discussion and a review of previous work, the reader is referred to Cairns, Blake, and Dowd (2006).

Continuous-time models have an important role to play in our understanding of how prices of mortality-linked securities will develop over time. However, the relative

\footnotetext{
${ }^{4}$ The Swiss Re mortality bond and the EIB longevity bond were the first to trade mortality risk exclusively. However, there have been previous issues of securities that packaged together several risks including mortality. The motivation for the issue of these securities goes beyond a desire purely to hedge mortality risk. A full discussion of these securities can be found in Cowley and Cummins (2005).
} 
intractability at the present time of such models is hindering their practical implementation. In this article, practical implementation of a model and statistical analysis are very much at the forefront of what we wish to achieve. Consequently, we choose to develop a model in discrete time and adopt an approach that is similar in vein to that of Lee and Carter (1992).

We propose a stochastic mortality model that we fit to UK mortality data and show how the calibrated model can be used to price mortality-linked financial instruments such as the EIB/BNP longevity bond. The model involves two stochastic factors. The first affects mortality at all ages in an equal manner, whereas the second has an effect on mortality that is proportional to age. We present empirical evidence that indicates that both these factors are needed to achieve a satisfactory empirical fit over the mortality term structure (that is, to model adequately historical mortality trends at different ages). The resulting model dynamics allow us to simulate cohort survival rates, thereby enabling us to model longevity risk, and to model other indices underlying alternative mortality-linked securities.

To price a mortality-linked security we adopt the risk-adjusted (or "risk-neutral") approach to pricing adopted by, for example, Milevsky and Promislow (2001) and Dahl (2004). Given the current dearth of market data, we propose a simple method for making the adjustment between real and risk-adjusted probabilities, which involves a constant market price for both longevity and parameter risk. The magnitude of this adjustment is established by estimating the market prices of these two risks implied by the proposed issue price of the EIB/BNP longevity bond.

Once a deep, liquid market in mortality-linked securities develops, however, we will be able to determine more reliable estimates of these market prices of risk and, indeed, to test that the hypothesis are constant.

The layout of this article is as follows. The "Model Specification" section outlines the model. The "Stochastic Mortality" section fits the model to English and Welsh mortality data, and discusses the plausibility of the fit. The next section presents some simulation results for the survivor index based on the calibrated model. Two alternative sets of simulation results are presented: first, results that do not take account of parameter uncertainty, and, second, results that do take account of such uncertainty. "The Price of Longevity Risk" discusses the premium that a life office or pension plan might be prepared to pay to lay off such risk-and uses this to show how the EIB/BNP bond might be priced in a risk-adjusted framework. Specifically, we focus on the market price of risk. It also presents some illustrative pricing results. "The Risk Premium on New Issues" shows how the earlier results might be used to price new longevity bonds with different terms to maturity and following different cohorts. "Sensitivity to the EIB Interest Rate" comments briefly on sensitivity of the results to changes in interest rates. In the following section, we discuss whether the market price of risk should be positive or negative, bearing in mind the requirements of different hedgers using different types on mortalitylinked contract. In the "Alternative Models" section, we give a brief discussion of alternative models including some comments on the cohort effect. The final section concludes. 


\section{MOdel Specification}

By analogy with interest-rate terminology, Cairns, Blake, and Dowd (2006) used the following notation for forward survival probabilities

$$
\begin{aligned}
p\left(t, T_{0}, T_{1}, x\right)= & \text { probability as measured at } t \text { that } \\
& \text { an individual aged } x \text { at time } 0 \text { and still alive at } T_{0} \\
& \text { survives until time } T_{1}>T_{0} .
\end{aligned}
$$

Let $I(u)$ represent the indicator process that is equal to 1 at time $u$ if the life aged $x$ at time 0 is still alive at time $u$, and 0 otherwise. Furthermore, let $\mathcal{M}_{u}$ be the filtration generated by the development of the mortality curve up to time $u .^{5}$ Then

$$
p\left(t, T_{0}, T_{1}, x\right)=\operatorname{Pr}\left(I\left(T_{1}\right)=1 \mid I\left(T_{0}\right)=1, \mathcal{M}_{t}\right) .
$$

Note that $p\left(t, T_{0}, T_{1}, x\right)=p\left(T_{1}, T_{0}, T_{1}, x\right)$ for all $t \geq T_{1}$, since the observation period $\left(T_{0}, T_{1}\right]$ is then past and not subject to any further uncertainty.

For simplicity in this exposition, we will define $\tilde{p}(t, x)=p(t+1, t, t+1, x)$ to be the realized survival probability for the cohort aged $x$ at time 0 . Additionally, define the realized mortality rate $\tilde{q}(t, x)=1-\tilde{p}(t, x)$.

In this article, we adopt the following $\operatorname{model}^{6}$ for the mortality curve:

$$
\tilde{q}(t, x)=1-p(t+1, t, t+1, x)=\frac{e^{A_{1}(t+1)+A_{2}(t+1)(x+t)}}{1+e^{A_{1}(t+1)+A_{2}(t+1)(x+t)}} .
$$

In this equation, $A_{1}(u)$ and $A_{2}(u)$ are stochastic processes that are assumed to be measurable at time $u$. An example of a mortality curve is given in Figure 1. This graph shows the ungraduated mortality rates above the age of 60 for England \& Wales males in $2002^{7}$ along with the fitted curve (fitted using least squares applied to (1)). The fit is clearly very good. Simpler parametric curves can also be fitted (for example, $q_{y}=$ $a^{A_{1}+A_{2} y}$ ) but the chosen curve gives a significantly better fit, especially for higher ages.

\section{StOchastic Mortality}

Estimated values for $A_{1}(t)$ and $A_{2}(t)$ for the years 1961-2002 are plotted in Figure $2 .{ }^{8}$ These results show a clear trend in both series. The downward trend in $A_{1}(t)$ reflects general improvements in mortality over time at all ages. The increasing trend in $A_{2}(t)$ means that the curve is getting slightly steeper over time: that is, mortality improvements have been greater at lower ages. There were also changes in the trend

\footnotetext{
${ }^{5}$ That is, $\mathcal{M}_{u}$ represents the history of the mortality curve up to time $u$.

${ }^{6}$ This is a special case of what are known as Perks models: see, for example, Perks (1932) or Benjamin and Pollard (1993).

${ }^{7}$ Available from the Government Actuary's Department website, www.gad.gov.uk.

${ }^{8}$ For each $t, A_{1}$ and $A_{2}$ were estimated using least squares by transforming the ungraduated mortality rates from $q_{y}$ to $\log q_{y} / p_{y}=A_{1}+A_{2} y+$ error.
} 


\section{FIGURE 1}

Ungraduated Mortality Rates Above the Age of 60 for England and Wales Males for the Year 2002 (dots) and Fitted Curve $e^{A_{1}+A_{2} y} /\left(1+e^{A_{1}+A_{2} y}\right)$ for $A_{1}=-10.95$ and $A_{2}=0.1058$

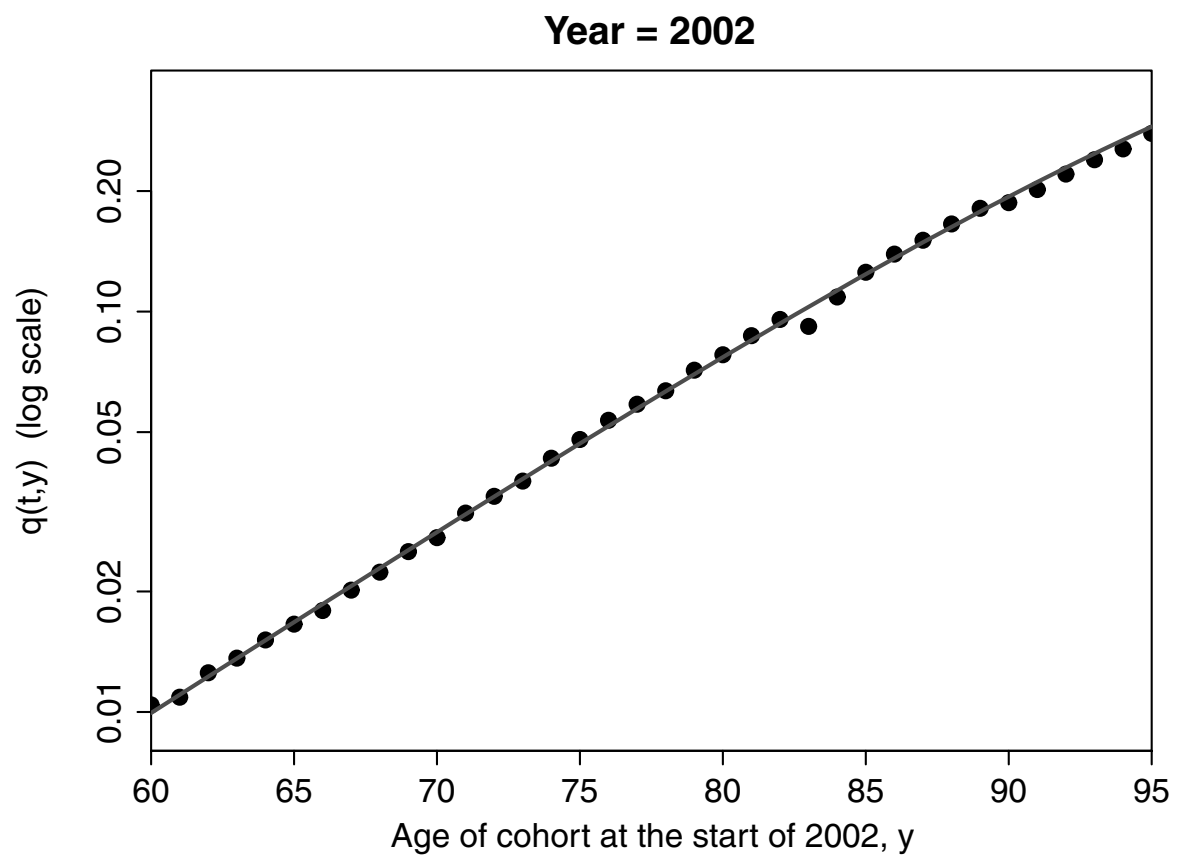

and in the volatility of both series. To make forecasts of the future distribution of $A(t)=\left(A_{1}(t), A_{2}(t)\right)^{\prime}$, we will model $A(t)$ as a two-dimensional random walk with drift. Specifically,

$$
A(t+1)=A(t)+\mu+C Z(t+1),
$$

where $\mu$ is a constant $2 \times 1$ vector, $C$ is a constant $2 \times 2$ upper triangular matrix ${ }^{9}$ and $Z(t)$ is a two-dimensional standard normal random variable. If we use data from 1961 to 2002 (41 observations of the differences) we find that

$$
\hat{\mu}=\left(\begin{array}{l}
-0.0434 \\
0.000367
\end{array}\right), \text { and } \hat{V}=\hat{C} \hat{C}^{\prime}=\left(\begin{array}{cc}
0.01067 & -0.0001617 \\
-0.0001617 & 0.000002590
\end{array}\right)
$$

\footnotetext{
${ }^{9}$ There are infinitely many matrices $C$ that satisfy $V=C C^{\prime}$, but the choice of $C$ makes no difference to our analysis. Provided the entries of $C$ are all real valued, $C C^{\prime}$ is always positive semidefinite. The restriction of $C$ to an upper-triangular form means that $C$ is straightforward to derive from $V$ and that this (Cholesky) decomposition is unique.
} 


\section{FigURE 2}

Estimated Values of $A_{1}(t)$ (Left-Hand Panel) and $A_{2}(t)$ (Right-Hand Panel) in Equation (1) from 1961 to 2002 for England and Wales Males
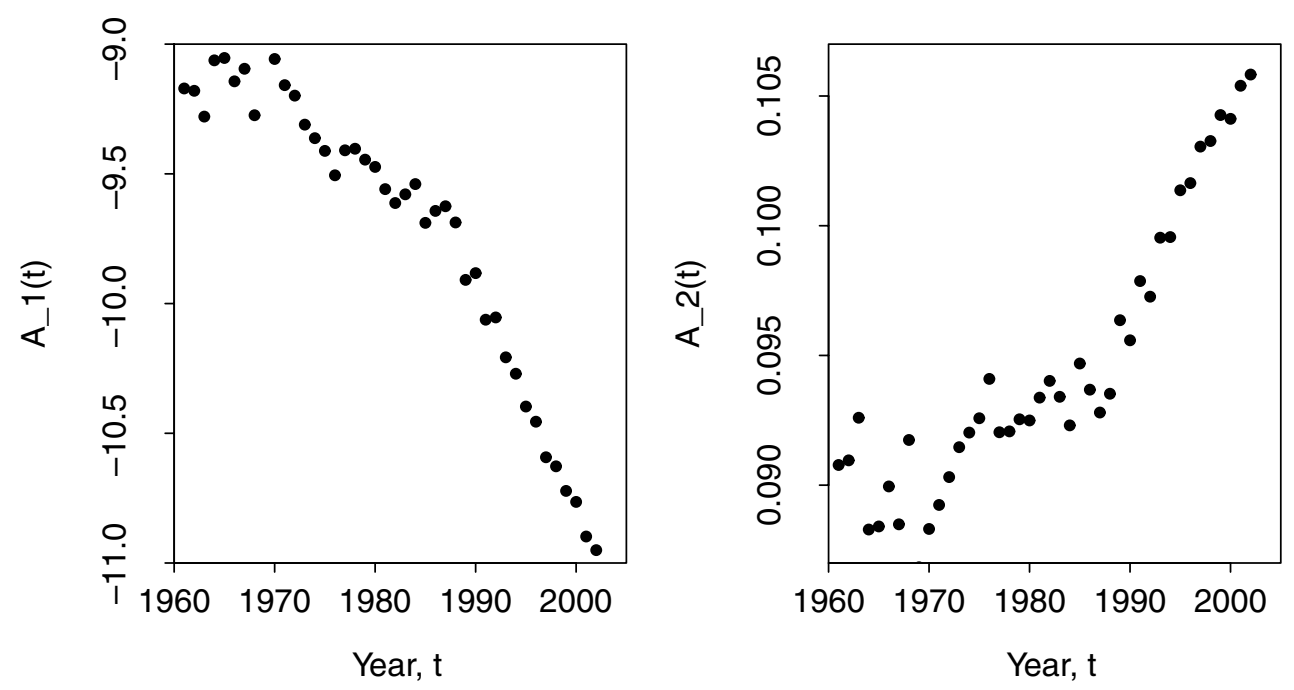

If, on the other hand, we use data from 1982 to 2002 only (20 observations) then we find that

$$
\hat{\mu}=\left(\begin{array}{c}
-0.0669 \\
0.000590
\end{array}\right), \text { and } \hat{V}=\hat{C} \hat{C}^{\prime}=\left(\begin{array}{cc}
0.00611 & -0.0000939 \\
-0.0000939 & 0.000001509
\end{array}\right) \text {. }
$$

These results show a steepening of trends after 1982, with $\mu_{1}$ and $\mu_{2}$ both becoming larger in magnitude. They also show that the volatilities in the later period were notably smaller than in the earlier period.

An important criterion for a good mortality model (see Cairns, Blake, and Dowd, 2006, for a discussion) requires the model and its parameter values to be biologically reasonable. ${ }^{10}$ The negative value for $\mu_{1}$ indicates generally improving mortality, with this improvement strengthening after 1982. The positive value for $\mu_{2}$ means that mortality rates at higher ages are improving at a slower rate. Indeed, above the very high age of 113, the model predicts deteriorating mortality. ${ }^{11}$ This might be perceived to be an undesirable feature of our model, but because this crossover point is at such a high age it is not felt to be a serious problem here as the number of lives involved is very low.

${ }^{10}$ Experts in mortality will hold certain subjective views on how mortality rates might evolve over time or how mortality rates at different ages ought to relate to one another. Examples of such criteria include: a requirement that the mortality curve in each calendar year is increasing with age at higher ages; and models that give rise to a strictly positive probability of immortality should be ruled out. Many experts would agree with these criteria but others might not.

${ }^{11}$ In other words, the mortality rate at ages $>113$ is rising over time rather than lowering. 
An additional criterion for biological reasonableness is that, in any given year in the future, we should normally see mortality rates for older cohorts that are higher than those for younger cohorts (that is, for fixed $t, \tilde{q}(t, x)$ should be an increasing function of $x$ ). This criterion requires $A_{2}(t)$ to remain positive. In our model $A_{2}(t)$ could, theoretically, become negative, but the positive value for $\mu_{2}$ and the initial value for $A_{2}$ in 2002 of 0.1058 means that $A_{2}(t)$ is very unlikely to do so. So the possibility of a negative $A_{2}(t)$ is of little significance and for all practical purposes our model be regarded as satisfying this second criterion of biological reasonableness as well.

\section{Cohort Dynamics}

In subsequent sections we will focus on the dynamics of a survivor index, $S(t)$. This is built up with reference to the mortality rates over time of one specific cohort, and it makes sense, therefore, to look at cohort dynamics within the context of our two-factor model. Investigating cohort dynamics also gives us the opportunity to make a further check on biological reasonableness.

In some contexts, following a cohort might mean analyzing the force of mortality and its dynamics over time. However, in the present article, we have chosen to work in discrete time, so we will consider the dynamics of $\tilde{q}(t, x)$ for a cohort aged $x$ at time 0 . It simplifies matters if we consider

$$
\begin{aligned}
\log \tilde{q}(t+1, x) / \tilde{p}(t+1, x)= & A_{1}(t+1)+A_{2}(t+1)(x+t+1) \\
= & (1, x+t+1)^{\prime}[A(t)+\mu+C Z(t+1)] \\
= & \log \tilde{q}(t, x) / \tilde{p}(t, x) \\
& +\left(\mu_{1}+\mu_{2}(x+t+1)+A_{2}(t)\right)+(1, x+t+1)^{\prime} C Z(t+1) .
\end{aligned}
$$

Now $A_{2}(t)$ is currently around 0.1058 and expected to increase slowly $\left(\mu_{2}>0\right)$. Furthermore, the standard deviation of $A_{2}(t)$ is very small over the time horizons we are likely to consider (for example, the standard deviation of $A_{2}(25)$ is 0.006 ). Thus $\mu_{1}+\mu_{2}+A_{2}(t)$ is initially positive and is expected to stay positive. As a consequence, the cohort will experience generally increasing rates of mortality with occasional falls in years when there is a large random mortality improvement across the board (that is, when $\left.(1, x+t+1)^{\prime} C Z(t+1) \ll 0\right)$.

\section{Simulation Results for The SURvivor Index S(t)}

A longevity bond of the type proposed by the EIB/BNP indexes coupon payments in line with a survivor index $S(t)$ for a specified cohort of individuals. ${ }^{12}$

We now wish to determine the distribution for $S(t)$ for the times $t=1,2, \ldots, 25$ that are relevant for the EIB/BNP bond. Even though the functional form for $\tilde{q}(t, x)$ is relatively simple, its distribution for $t>2$ is not analytically tractable, so we resort to Monte Carlo simulation and obtain the simulated $\tilde{q}(t, x)$ and $S(t)$ from simulations of the underlying process $A(t)$.

\footnotetext{
12 In the case of the EIB/BNP bond, the reference cohort is the set of all England and Wales males aged 65 in 2003. The method used to calculate $S(t)$ for this cohort is given in Appendix A.
} 


\section{Results with No Allowance for Parameter Uncertainty}

In our first experiment, we simulated the $A(t)$ according to Equation (2) using estimates for $\mu$ and $V$ based on data from 1961-2002 to 1982-2002. These parameter estimates were treated as if they were the true parameter values, implying that, to begin with, we ignore parameter uncertainty. The results are plotted in Figure 3. We can make the following observations:

- The solid curves plot the expected values of $S(t)$. Measured at time 0 , these represent the ex ante probabilities of survival from time 0 to time $t, p(0,0, t, 65)$ (which we refer to as spot survival probabilities). The mean trajectory based on data from 1982 to 2002 (bottom plot) is slightly higher than that in the upper plot (based on 1961-2002 data). This is because steepening trends in $A_{1}(t)$ and $A_{2}(t)$ in the 1982-2002 data (Figure 2) signal greater improvements in the future.

- The dashed curves in each plot show the 5th and the 95th percentiles of the distribution of $S(t)$. We can observe that the resulting $90 \%$ confidence interval is initially quite narrow but becomes quite wide by the 25-year time horizon (which is the maturity of the EIB/BNP longevity bond). We can also see that the confidence interval based on 1982-2002 data is a little narrower, reflecting the smaller values on the diagonal of $V$.

- The confidence interval for $S(t)$ grows in quite a different way from, say, that associated with an investment in equities. This point is best illustrated by looking at the variance of the logarithm of $S(t)$, as illustrated in Figure 4 . We can see that this is very low in the early years indicating that we can predict with reasonable certainty what mortality rates will be over the near future. However, after time 10 the variance starts to grow very rapidly (almost "exponentially"). This contrasts with equities where we would expect to see linear, rather than "exponential," growth in the variance if the price process follows geometric Brownian motion. The explanation for this variance growth is that the longer-term survival probabilities incorporate the compounding of year-by-year mortality shocks: the survival probability for year $t$ depends on shocks applied to mortality rates in each of the years 1 to $t$, and each individual shock affects survival probabilities in all subsequent years. ${ }^{13}$

\section{Results with Parameter Uncertainty}

We consider next the impact of parameter uncertainty. It is clear that we have a limited amount of data and so the parameter estimates above must inevitably be subject to some degree of uncertainty. We will analyze this using standard Bayesian methods. ${ }^{14}$

Recall that we have assumed that the process $A(t)$ is subject to i.i.d. multivariate normal shocks with mean $\mu$ and covariance matrix $V$. In the absence of any clear prior beliefs about the values of $\mu$ and $V$ we will use a non-informative prior distribution. A common prior for the multivariate normal distribution in which both $\mu$ and $V$ are unknown is the Jeffreys prior

$$
p(\mu, V) \propto|V|^{-3 / 2}
$$

\footnotetext{
${ }^{13}$ For further intuition, see Appendix C.

${ }^{14}$ For a general discussion of model and parameter risk, see Cairns, 2000.
} 


\section{FigURE 3}

Mean and Confidence Intervals for Projected Survival Probabilities Based on Data from 1961-2002 (Top Panel) or 1982-2002 (Bottom Panel). Each Plot Shows the Mean (Solid Curve) and the 5th and 95th Percentiles (Dashed Curves) of the Simulated Distribution of the Reference Index, $S(t)$, with No Allowance for Parameter Uncertainty.
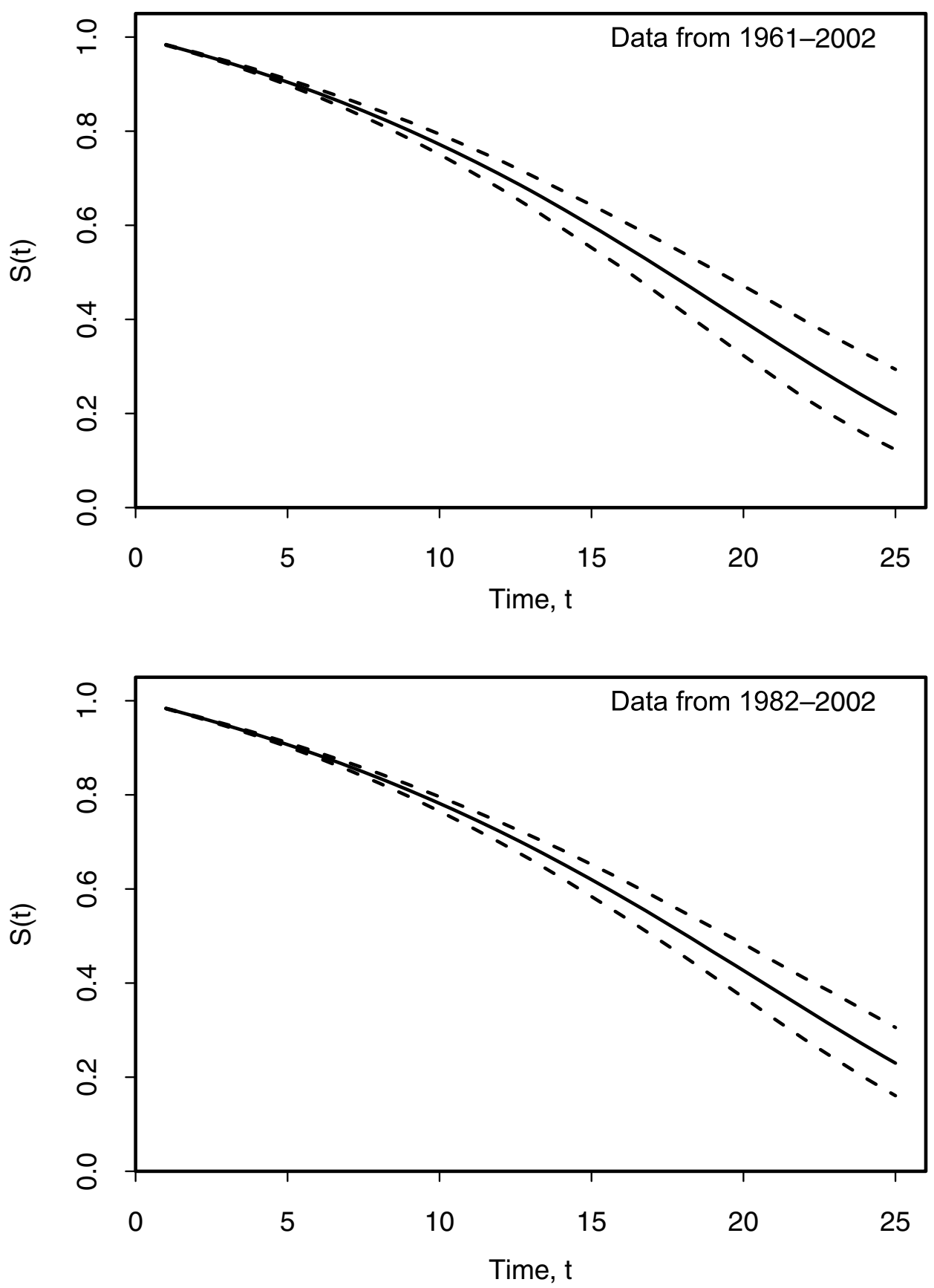


\section{FIGURE 4}

Plot of the Variance of $\log S(t)$ Using Data From 1961-2002 (Solid Curve) and from 1982-2002 (Dashed Curve), with No Allowance for Parameter Uncertainty

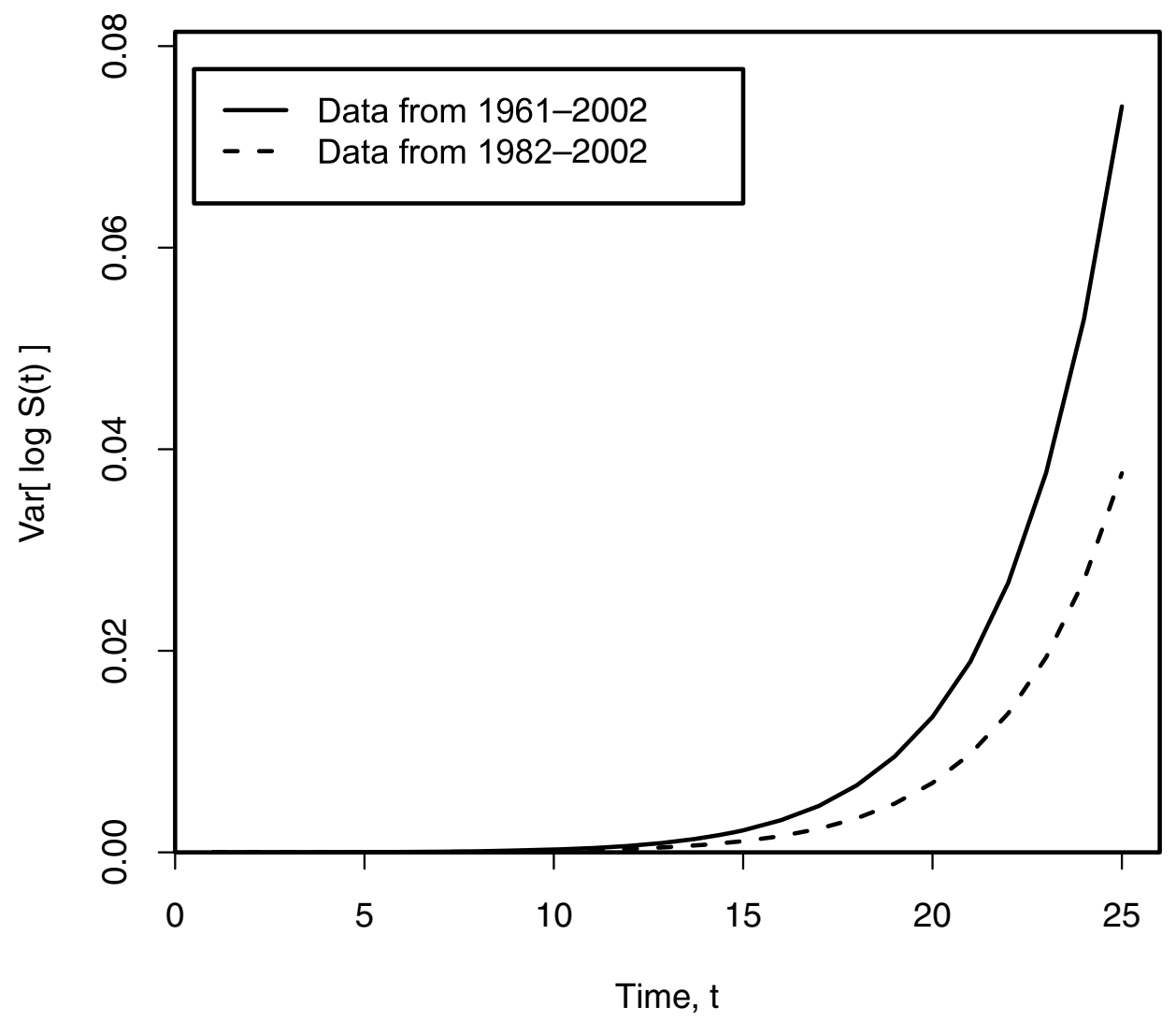

where $|V|$ is the determinant of the matrix $V .{ }^{15}$ With this prior and with $n$ observations $D=\{D(1), \ldots, D(n)\}$ (where $D(t)=A(t)-A(t-1)$ ), it is known that (see, for example, Gelman et al., 1995) the posterior distribution ${ }^{16}$ for $\mu, V \mid D$ is:

$$
\begin{aligned}
V^{-1} \mid D & \sim \operatorname{Wishart}\left(n-1, n^{-1} \hat{V}^{-1}\right) \\
\mu \mid V, D & \sim \operatorname{MVN}\left(\hat{\mu}, n^{-1} V\right), \\
\text { where } \hat{\mu} & =\frac{1}{n} \sum_{t=1}^{n} D(t) \\
\text { and } \hat{V} & =\frac{1}{n} \sum_{t=1}^{n}(D(t)-\hat{\mu})(D(t)-\hat{\mu})^{\prime} .
\end{aligned}
$$

\footnotetext{
${ }^{15}$ Since $V$ is strictly positive definite, its determinant is strictly positive.

${ }^{16}$ The Wishart distribution is a multivariate version of the Gamma or Chi-squared distribution. For details on how to simulate the joint Normal-Inverse-Wishart distribution, see Appendix B.
} 
In what follows, we will restrict ourselves to an analysis based on data from 1982 to 2002.

For each simulated sample path of $A(t)$, we simulate first $\mu$ and $V$ from the NormalInverse-Wishart distribution and use these values for the whole of that sample path. The results of these simulations can be seen in Figures 5 and 6. In Figure 5, we can see the impact of parameter uncertainty on the confidence interval: specifically that parameter uncertainty becomes much more significant as a source of uncertainty in $S(t)$ as $t$ increases. We can see that 25 years ahead parameter uncertainty accounts for about half of the uncertainty in $S(t) \cdot{ }^{17}$ In Figure 6, we plot the variance of $\log S(t)$, and the use here of a log scale allows us to see clearly that for smaller values of $t$ parameter uncertainty is much less important (that is, the difference between the two curves is quite small).

\section{The Price of Longevity Risk}

Now consider the price that a life office or pension fund might be prepared to pay to lay off its exposure to longevity risk. From Figures 3 to 6 we can infer that if premiums are to be paid in respect of each future year, the premium will be much larger for the 25 -year payment than, say, the 10 -year payment. Furthermore, a reasonable proportion of this premium might be in respect of the desire to eliminate exposure to parameter uncertainty.

\section{Pricing Using Risk-Adjusted Probability Measures}

We propose to specify the dynamics under a risk-adjusted pricing measure $Q$ that is equivalent to, in the probabilistic sense, the current real-world measure (which we shall refer to as $P) .{ }^{18}$ The measure $Q$ is also commonly referred to as the risk-neutral measure ${ }^{19}$ or as an equivalent-martingale measure.

Recall that we worked earlier with the following dynamics under $P$ :

$$
A(t+1)=A(t)+\mu+C Z(t+1)
$$

where $Z(t+1)$ is a standard 2-dimensional normal random variable under $P$.

${ }^{17}$ That is, at $t=25$ the size of the gap on the log scale between the solid and dashed lines equates to a ratio of about 2 between the variances.

${ }^{18}$ An alternative way of generating risk-adjusted measures is to use the Wang transform (Wang, $2000,2002,2003)$. These distort the distributions of each of the $S(t)$ random variables. Examples of its application to longevity bonds and other mortality-linked securities include Lin and Cox (2005); Denuit, Devolder and Goderniaux (2004); Cox, Lin and Wang (2005); and Dowd et al. (2006).

${ }^{19} \mathrm{In}$ an incomplete market, the term risk-neutral is vague, but is used to convey the point that expected returns over the short term under $Q$ are equal to the short-term risk-free rate of interest. At the present time, we are very far from having a complete market in which all contingent claims can be replicated using dynamic hedging strategies. This means that the risk-adjusted measure $Q$ is not unique. 


\section{Figure 5}

Confidence Intervals for Projected Survival Probabilities Based on Data from 1982 to 2002. Confidence Intervals Are Shown Excluding Parameter Uncertainty (Thin Dotted Curves) and Including Parameter Uncertainty (Thick Dashed Curves). The Mean Trajectories (Thin and Thick Solid Curves) for the Two Cases Are Overlapping.

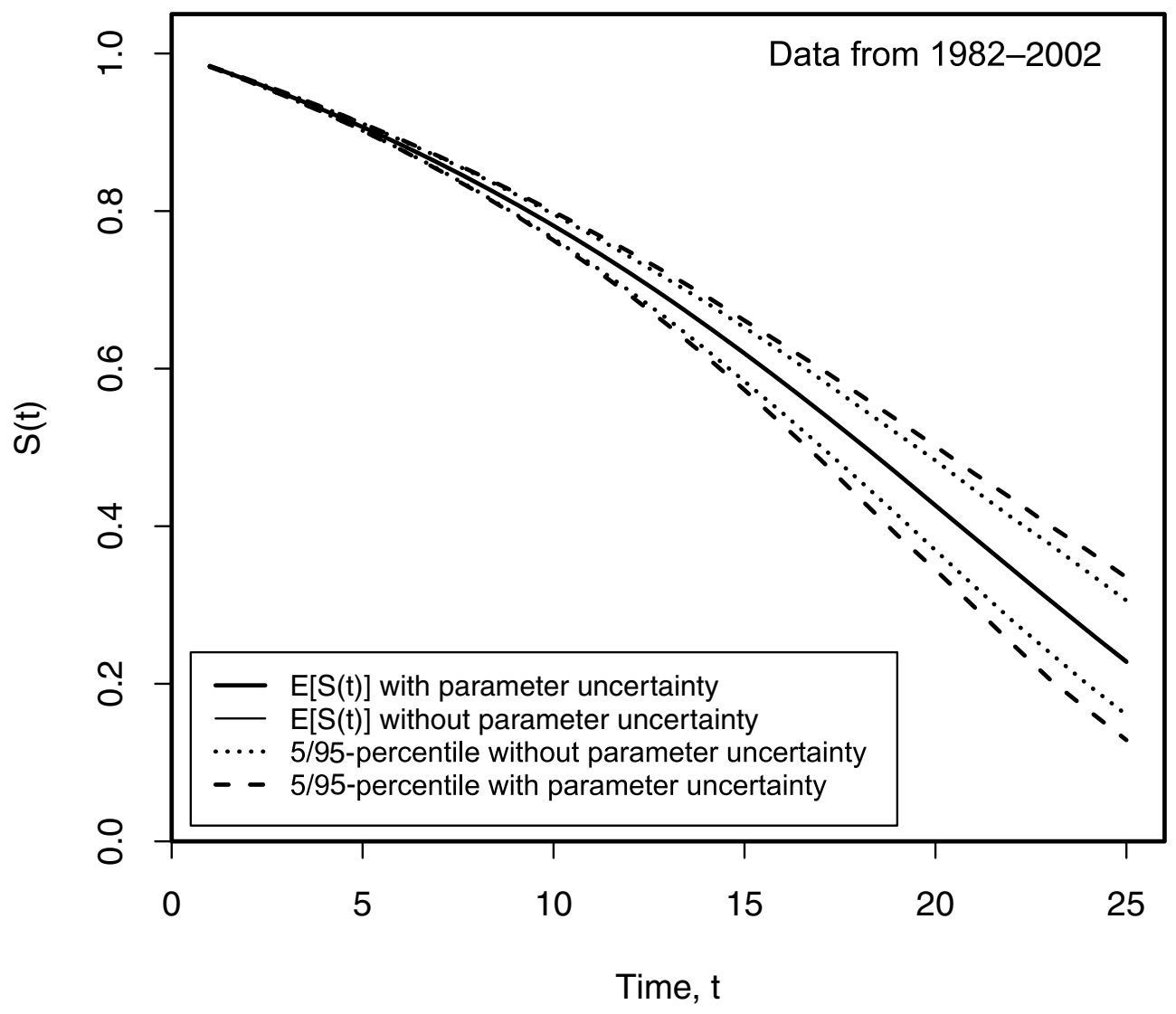

Under the risk-adjusted measure $Q(\lambda)$ we propose ${ }^{20}$ that:

$$
\begin{aligned}
& \begin{aligned}
A(t+1) & =A(t)+\mu+C(\tilde{Z}(t+1)-\lambda) \\
& =A(t)+\tilde{\mu}+C \tilde{Z}(t+1), \\
\text { where } \tilde{\mu} & =\mu-C \lambda .
\end{aligned}
\end{aligned}
$$

${ }^{20}$ Modelling in discrete time means that there are infinitely-many equivalent measures to choose from with different means, variances and covariances for $Z(t+1)$. However, we choose to restrict ourselves to ones that have a constant market price of risk, that preserve the variance-covariance structure of $Z(t+1)$, and that preserve the assumption of bivariate normality. The latter assumptions lead to consistency between the discrete-time model and the continuous-time diffusion model. 


\section{Figure 6}

Plot of the Variance (on a Log Scale) of log $S(t)$ Using Data from 1982 to 2002. The Variance has been Calculated Excluding Parameter Uncertainty (Dashed Curves) and Including Parameter Uncertainty (Solid Curves).

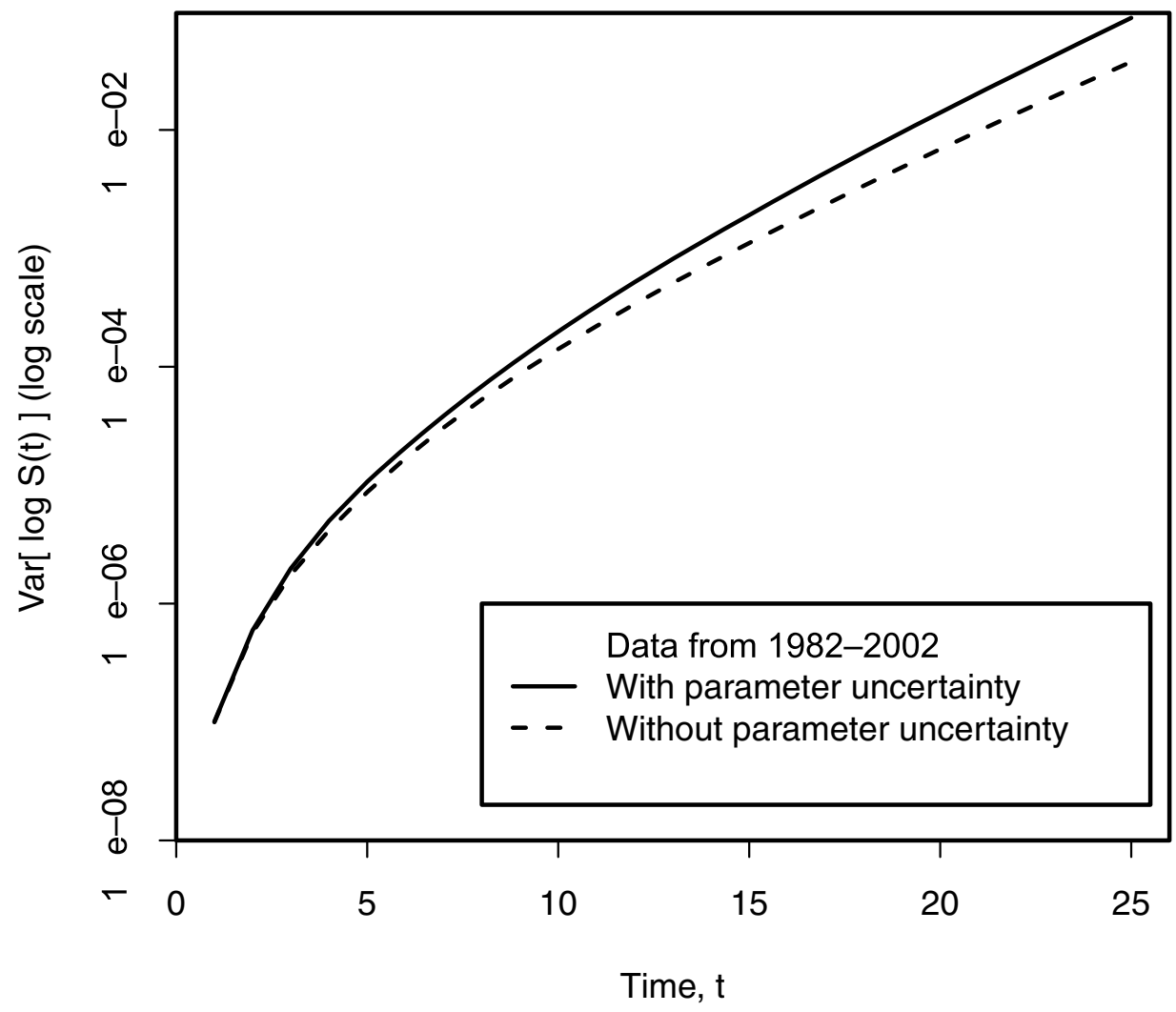

In this equation $\tilde{Z}(t+1)$ is a standard two-dimensional normal random variable ${ }^{21}$ under $Q$. The vector $\lambda=\left(\lambda_{1}, \lambda_{2}\right)$ represents the market prices of longevity risk associated with the processes $Z_{1}(t)$ and $Z_{2}(t)$, respectively. Under the chosen decomposition for the matrix $C$ (upper triangular), $\lambda_{1}$ is associated with level shifts in mortality (specifically $\log q / p$ ), while $\lambda_{2}$ is associated with a tilt in $\log q / p$. We assume (as part of our model) that $\lambda$ is constant rather than time dependent: indeed it is difficult to propose anything more sophisticated for $\lambda$ in the absence of any market price data.

We can make the following points about $Q(\lambda)$ :

- Complete market models such as the Black-Scholes option-pricing model force upon us a unique choice of measure $Q$. In contrast, here we have an incomplete market, and a range of possibilities for $Q(\lambda)$.

- If there exists some form of market in mortality-linked securities then the choice of $Q(\lambda)$ needs to be consistent with this (limited) market information (so that theoretical prices under $Q(\lambda)$ match observed market prices).

${ }^{21}$ That is, $\tilde{Z}(t+1)=\left(\tilde{Z}_{1}(t+1), \tilde{Z}_{2}(t+1)\right)^{\prime}$ where $\tilde{Z}_{1}(1), \tilde{Z}_{1}(2), \ldots$ and $\tilde{Z}_{2}(1), \tilde{Z}_{2}(2), \ldots$ are independent sequences of i.i.d. $N(0,1)$ random variables under $Q(\lambda)$. 
- Beyond these restrictions, the choice of $Q(\lambda)$ becomes a modelling assumption. Thus, here we have postulated that the market price of risk, $\lambda$, might be constant over time (in the same way that the market price of risk is normally assumed to be constant in the Black-Scholes model). ${ }^{22}$

- The assumptions embedded in $Q(\lambda)$ form a testable hypothesis. However, the assumptions can only be tested once a market develops in a range of mortality-linked securities and with sufficient liquidity over time that historical price data can be used to test if the assumption that the market price of risk is constant or time varying.

\section{Example: The EIB/BNP Longevity Bond}

As an example, consider the 25-year EIB/BNP longevity bond announced in November 2004, with an issue price based on a yield of 35 basis points below LIBOR. The appropriate starting point is the EIB curve for conventional fixed-interest bonds issued typically at 15 basis points below LIBOR. This means that the new longevity bond was priced at 20 basis points below standard EIB rates. This spread below standard EIB rates will be denoted by $\delta$ in the equations that follow. We will now make the following assumptions:

- The projected survival rates used in the pricing of the bond (in the case of the EIB/BNP bond, this is the projection made by the UK Government Actuary's Department) are unbiased estimates at time 0 under the real-world measure $P$ of the survival rates.

- The spread of 20 basis points below the standard EIB curve is accounted for entirely by the market price of longevity risk.

- The development of mortality rates over time is independent of the dynamics of the interest-rate term structure over time. ${ }^{23}$

We will refer to $\hat{S}(0, T)$ as the survivor index based upon the latest GAD projections available at time $0 .{ }^{24}$ Assumption 1 implies that $\hat{S}(0, T)=E_{P}\left[S(T) \mid \mathcal{M}_{0}\right]$.

Next, let us refer to $P(0, T)$ as the price at time 0 of a fixed-principle zero-coupon bond issued by the EIB that pays 1 at time T. The basis declared by the EIB and BNP for the initial price of the bond was $V(0)=\sum_{T=1}^{25} P(0, T) e^{\delta T} \hat{S}(0, T)$, where $\delta$ is the spread

${ }^{22}$ In the case of both the present model and the Black-Scholes model, it would seem appropriate that the market price be allowed to vary in a stochastic fashion over the long term, 25 years, of the contract. However, in the equity-modelling literature there seems to be little consensus on the dynamics of the market price of risk. If we combine this observation with the absence of any historical market data on mortality-linked securities we conclude that there is little point in attempting to model the market-price of risk as a dynamic process.

${ }^{23}$ This is a very useful simplifying assumption which we believe to be a reasonable one for relatively short horizons under normal conditions. However, we recognize anecdotal evidence (see, for example, Miltersen and Persson, 2005) that over the very long run the term structure of interest rates will be influenced by the relative size of the capital stock to that of the population, and the latter might be influenced by mortality (as well as fertility) dynamics. Also in the short run, we recognize that a catastrophe that affects the size of the population (such as nuclear war) will also affect interest rates.

${ }^{24}$ Values for the $\hat{S}(0, T)$ are specified in the offer document issued by BNP Paribas. 
(expressed as a continuously compounding rate) below the EIB curve used in pricing the bond..$^{25}$ Given assumption 1 this is equivalent to ${ }^{26}$

$$
V(0)=\sum_{T=1}^{25} P(0, T) e^{\delta T} E_{P}\left[S(T) \mid \mathcal{M}_{0}\right] .
$$

Values for $E_{P}\left[S(T) \mid \mathcal{M}_{0}\right]$ based upon parameters in (4) and without parameter uncertainty are given in Table 1, column 1 . For example, if we assume that implied EIB zero-coupon prices are given by $P(0, T)=1.04^{-T}$ and if we set $\delta=0.0020$ and $\lambda=(0,0)^{\prime}$ then we find that the price at issue of the bond on this contractual basis (Equation 7) is $V(0)=11.442$.

The risk-adjusted approach to pricing assumes that (exploiting assumption 3 above)

$$
V_{\lambda}(0)=\sum_{T=1}^{25} P(0, T) E_{Q(\lambda)}\left[S(T) \mid \mathcal{M}_{0}\right]
$$

A comparison of Equations (7) and (8) shows that $\delta$ can be interpreted as an average risk premium per annum. We shall see later that this risk premium will depend upon the term of the bond and on the initial age of the cohort being tracked.

We can now ask the question: What values for the market prices of risk $\lambda_{1}$ and $\lambda_{2}$ satisfy $V_{\lambda}(0)=V(0)$ ? Put another way, under what circumstances does the risk-adjusted price (Equation 8) match the issue price quoted in the contract (Equation 7)?

With no parameter uncertainty, and $\delta=0$ we found that we could obtain $V_{\lambda}(0)=$ 11.442 with $\left(\lambda_{1}, \lambda_{2}\right)=(0.375,0)$ and $(0,0.316)$. For these two values for $\lambda$ the values for $E_{Q(\lambda)}\left[S(t) \mid \mathcal{M}_{0}\right]$ are given in Table 1 columns 3 and 4 . In column 5, we have also given an intermediate value for $\lambda$ between these two extremes. ${ }^{27}$ Here we can achieve $V_{\lambda}(0)=V(0)$ with $\lambda_{1}=\lambda_{2}=0.175 .^{28,29}$

${ }^{25}$ We do not know what the pricing convention for the bond will be after issue. However, it seems plausible that it will also be of the form (for integer $t$ ) $V(t)=\sum_{T=t+1}^{25}$ $P(t, T) e^{\delta(t)(T-t)} \hat{S}(0, T)$ : that is, still with reference to the initial estimate $\hat{S}(0, T)$, and with reference to an easily-observable zero-coupon curve at time $t$.

${ }^{26}$ Strictly, the $P(0, T)$ in Equation (7) should be LIBOR-implied discount factors, $P_{L}(0, T)$, in combination with $\delta=0.0035$ as stated in the contract, while the $P(0, T)$ in Equation (8) should be the EIB-implied discount factors, $P_{E}(0, T)$. However, as stated above, the approximate relationship between the two is $P_{E}(0, T)=P_{L}(0, T) e^{0.0015 T}$, which leads us to the given form in Equation (7) with $\delta=0.0020$.

${ }^{27}$ This can be found by fixing first the value for $\lambda_{1}$ and then solving for $\lambda_{2}$.

${ }^{28} \mathrm{In}$ fact, the set of values for $\left(\lambda_{1}, \lambda_{2}\right)$ that gives a price of 11.442 is approximately linear running from $(0.375,0)$ to $(0,0.316)$. A straight line between the two end points would pass through $(0.171,0.171)$ rather than $(0.175,0.175)$.

${ }^{29}$ It is tempting to think that $\left(\lambda_{1}, \lambda_{2}\right)=(0.375,0)$ and $(0,0.316)$ represent the extreme values for the market price of longevity risk. This might be true for $\left(\lambda_{1}, \lambda_{2}\right)=(0,0.316)$ if the demand for such assets is coming from annuity providers. However, if the market is dominated by life offices hedging short-term catastrophic mortality risk in their term-assurance portfolios, then $\lambda_{1}$ might, in fact, be negative. Similarly, $\lambda_{2}$ might be negative if longevity risk at ages below 60 presents the greatest risk to annuity providers. We have more to say on this subject in the section titled "The Sign of the Market Price of Risk." 


\section{TABLE 1}

Longevity Bond Expected Cashflows Under the Risk-Neutral Measure $Q(\lambda)$ : $E_{\mathrm{Q}(\lambda)}\left[S(t) \mid \mathcal{M}_{0}\right]$ and Market Prices Under Various Assumptions for the Market Prices of Longevity and Parameter Risk

\begin{tabular}{|c|c|c|c|c|c|c|c|}
\hline & \multicolumn{7}{|c|}{ Column Reference } \\
\hline & 1 & 2 & 3 & 4 & 5 & 6 & 7 \\
\hline Parameter uncertainty: & $\mathrm{N}$ & Y & $\mathrm{N}$ & $\mathrm{N}$ & $\mathrm{N}$ & Y & Y \\
\hline$\lambda_{1}$ & 0 & 0 & 0.375 & 0 & 0.175 & 0 & 0 \\
\hline$\lambda_{2}$ & 0 & 0 & 0 & 0.316 & 0.175 & 0 & 0 \\
\hline$\lambda_{3}$ & - & 0 & - & - & - & 1.684 & 0 \\
\hline$\lambda_{4}$ & - & 0 & - & - & - & 0 & 1.419 \\
\hline$t$ & \multicolumn{7}{|c|}{$E_{Q(\lambda)}\left[S(t) \mid \mathcal{M}_{0}\right]$} \\
\hline 1 & 0.9836 & 0.9836 & 0.9837 & 0.9836 & 0.9836 & 0.9837 & 0.9836 \\
\hline 2 & 0.9661 & 0.9661 & 0.9664 & 0.9662 & 0.9663 & 0.9664 & 0.9662 \\
\hline 3 & 0.9475 & 0.9475 & 0.9482 & 0.9477 & 0.9479 & 0.9482 & 0.9477 \\
\hline 4 & 0.9278 & 0.9278 & 0.9289 & 0.9281 & 0.9285 & 0.9289 & 0.9281 \\
\hline 5 & 0.9068 & 0.9068 & 0.9086 & 0.9074 & 0.908 & 0.9086 & 0.9074 \\
\hline 6 & 0.8845 & 0.8845 & 0.8872 & 0.8856 & 0.8863 & 0.8872 & 0.8856 \\
\hline 7 & 0.861 & 0.8609 & 0.8646 & 0.8626 & 0.8635 & 0.8646 & 0.8626 \\
\hline 8 & 0.836 & 0.8359 & 0.8408 & 0.8384 & 0.8395 & 0.8407 & 0.8383 \\
\hline 9 & 0.8095 & 0.8095 & 0.8157 & 0.8129 & 0.8142 & 0.8156 & 0.8129 \\
\hline 10 & 0.7816 & 0.7815 & 0.7893 & 0.7862 & 0.7877 & 0.7892 & 0.7861 \\
\hline 11 & 0.7522 & 0.752 & 0.7616 & 0.7583 & 0.7599 & 0.7615 & 0.7582 \\
\hline 12 & 0.7213 & 0.721 & 0.7326 & 0.7292 & 0.7308 & 0.7325 & 0.729 \\
\hline 13 & 0.6888 & 0.6885 & 0.7023 & 0.6989 & 0.7004 & 0.7021 & 0.6987 \\
\hline 14 & 0.6548 & 0.6545 & 0.6707 & 0.6675 & 0.6689 & 0.6704 & 0.6672 \\
\hline 15 & 0.6195 & 0.6191 & 0.6378 & 0.635 & 0.6362 & 0.6374 & 0.6346 \\
\hline 16 & 0.5828 & 0.5823 & 0.6036 & 0.6015 & 0.6024 & 0.6032 & 0.6011 \\
\hline 17 & 0.5448 & 0.5443 & 0.5684 & 0.5672 & 0.5676 & 0.5679 & 0.5667 \\
\hline 18 & 0.5059 & 0.5052 & 0.5321 & 0.5321 & 0.532 & 0.5315 & 0.5316 \\
\hline 19 & 0.4661 & 0.4654 & 0.495 & 0.4965 & 0.4957 & 0.4944 & 0.4959 \\
\hline 20 & 0.4258 & 0.4251 & 0.4573 & 0.4606 & 0.459 & 0.4566 & 0.4599 \\
\hline 21 & 0.3853 & 0.3847 & 0.4191 & 0.4245 & 0.422 & 0.4185 & 0.4238 \\
\hline 22 & 0.345 & 0.3445 & 0.3809 & 0.3885 & 0.3851 & 0.3803 & 0.3879 \\
\hline 23 & 0.3054 & 0.305 & 0.3428 & 0.353 & 0.3486 & 0.3424 & 0.3524 \\
\hline 24 & 0.2667 & 0.2668 & 0.3054 & 0.318 & 0.3128 & 0.3052 & 0.3177 \\
\hline 25 & 0.2297 & 0.2302 & 0.2689 & 0.2841 & 0.278 & 0.269 & 0.284 \\
\hline \multicolumn{8}{|l|}{ Price } \\
\hline$\delta=0$ & 11.240 & 11.237 & 11.442 & 11.442 & 11.442 & 11.439 & 11.439 \\
\hline$\delta=0.0020$ & 11.442 & 11.439 & - & _- & - & - & \\
\hline
\end{tabular}

We next introduce parameter uncertainty into the analysis. We first simulate under $P$ with full parameter uncertainty and the values for $E_{Q(\lambda)}\left[S(t) \mid \mathcal{M}_{0}\right]$ are given in Table 1, in column 2 with $\lambda=(0,0,0,0)^{\prime}$. We have seen in Figure 6 that parameter uncertainty presents a significant risk to annuity providers. It follows that they will be prepared to pay a premium to reduce this risk in the same way that they are prepared to pay to reduce the impact of longevity risk. 
In this analysis, we concentrate on introducing a market price of risk for the mean $\mu$. In the case of no parameter uncertainty we assume that $\mu=\hat{\mu}$. With parameter uncertainty, the basic model (see Appendix B) simulates first $V$, then calculates the upper-triangular matrix $C$ that satisfies $V=C C^{\prime}$, and then simulates

$$
\mu=\hat{\mu}+n^{-1 / 2} C Z_{\mu}
$$

where $Z_{\mu}$ is a standard bivariate normal random variable. Once again we have to identify possible equivalent measures. We propose here a similar restriction that, under the equivalent measure, $Z_{\mu}$ is still bivariate normal with unit variances but a shifted mean. Thus we now simulate $\mu$ with reference to two additional market prices of risk $\lambda_{3}$ and $\lambda_{4}$ :

$$
\mu=\hat{\mu}+n^{-1 / 2} C\left(\tilde{Z}_{\mu}-\lambda_{\mu}\right)=\tilde{\mu}+n^{-1 / 2} C \tilde{Z}_{\mu}
$$

where $\lambda_{\mu}=\left(\lambda_{3}, \lambda_{4}\right)^{\prime}$ and $\tilde{\mu}=\hat{\mu}-n^{-1 / 2} C \lambda_{\mu}$.

We now have four market prices of risk to play with to match the single price derived by discounting expected cashflows under $P$ at EIB minus 20 basis points. With parameter uncertainty included, the expected cashflows under $P$ change very slightly (see Table 1 , column 2), as does the price of $V(0)=11.439$. The values for $\lambda_{1}$ and $\lambda_{2}$ required to match this price are essentially unchanged from the values that were determined before (Table 1, columns 3 and 4) and are consequently not repeated in the table. The required values for $\lambda_{3}$ and $\lambda_{4}$ were, respectively, 1.684 and 1.419 , with the corresponding values for $E_{Q(\lambda)}\left[S(t) \mid \mathcal{M}_{0}\right]$ quoted in Table 1, columns 6 and 7.

For the various cases presented in Table 1 we have plotted in Figure 7 the expected value under $P$ or $Q(\lambda)$ of $S(t)$ for $t=1, \ldots, 25$. This plot helps us to analyze the impact of using the different measures and, in particular, to see where most of the additional value in the longevity bond resides. The expected values in the upper plot show us two things. First, the inclusion of parameter uncertainty has almost no effect on the expected values under $P$. Second, the expected values under the different $Q(\lambda)$ measures look similar, and all show up the largest differences compared with the $P$ measure near $t=25$.

The lower plot in Figure 7 allows us to differentiate more easily between the different $Q(\lambda)$ measures. Here we have plotted the expected risk premium per annum on a zero-coupon longevity bond that makes a single payment of $S(t)$ at time $t$ that is held from time 0 through to maturity at $t$. This is calculated by converting the ratio of two expected values into an additional rate of return per annum as follows:

$$
\frac{1}{t} \log \left(\frac{E_{Q(\lambda)}\left[S(t) \mid \mathcal{M}_{0}\right]}{E_{P}\left[S(t) \mid \mathcal{M}_{0}\right]}\right)
$$

From this lower plot we can see that the level of the risk premium depends to some extent on the choice of $Q(\lambda)$. However, we can note from both Figure 7 and Table 1 that cases 3 and 6 produce very similar results and that cases 4 and 7 also produce very 


\section{FIGURE 7}

Top Panel: Expected Value of $S(t)$ Under Different Probability Measures. Values for the Market Prices of Risk in the Six Cases Considered Are Given in the Legend; $p=0$ Means without Parameter Uncertainty and $p=1$ Means with Parameter Uncertainty in Both $\mu$ and $V$. Bottom Panel: Average Risk Premium Per Annum Is Defined as $\log \left\{E_{Q(\lambda)}[S(t)] / E_{P}[S(t)]\right\} / t$ on a Zero-Coupon Longevity Bond Over the Full Term to Maturity. Different Line Types Are Defined in the Top Plot.

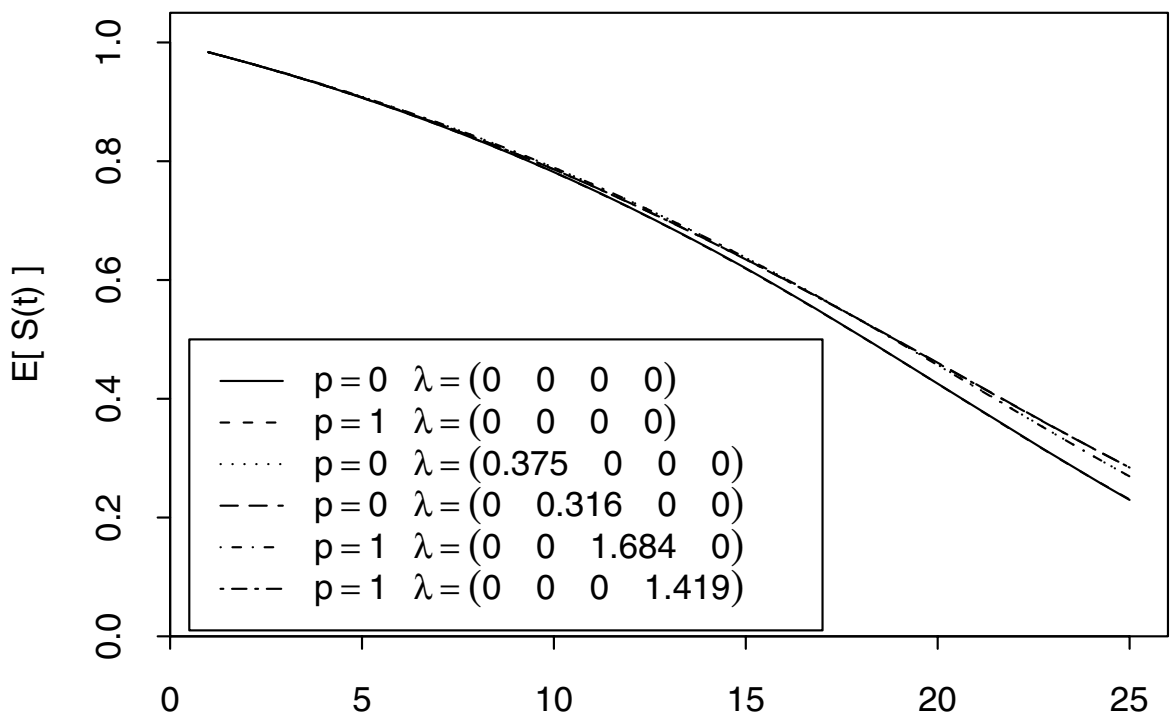

Maturity, $\mathrm{t}$

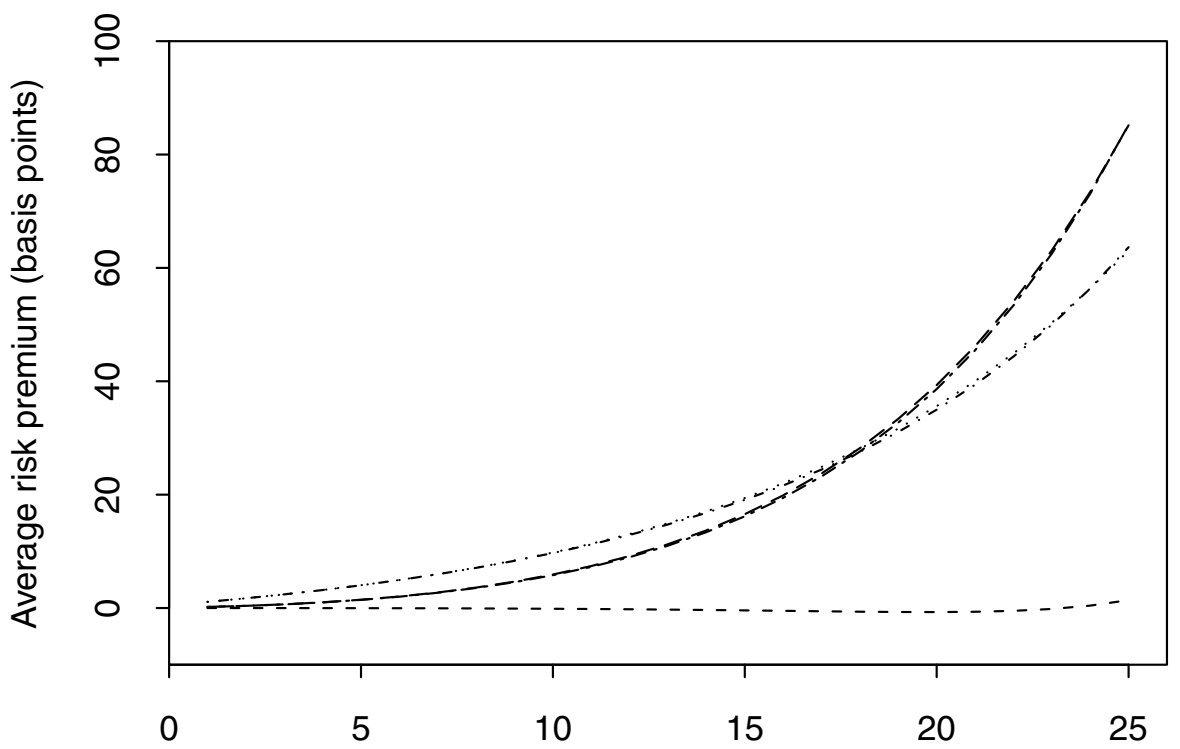

Maturity, $\mathrm{t}$ 
similar results. ${ }^{30}$ The reason for these similarities is not, in fact, difficult to explain. Recall that we have

$$
\begin{aligned}
\mu & =\hat{\mu}-n^{-1 / 2} C \lambda_{\mu}+n^{-1 / 2} C \tilde{Z}_{\mu} \\
\text { and } A(t+1) & =A(t)+\mu-C \lambda+C \tilde{Z}(t+1) \\
& =A(t)+\hat{\mu}-n^{-1 / 2} C \lambda_{\mu}-C \lambda+n^{-1 / 2} C \tilde{Z}_{\mu}+C \tilde{Z}(t+1) \\
& =A(t)+\hat{\mu}-C\left(n^{-1 / 2} \lambda_{\mu}+\lambda\right)+n^{-1 / 2} C \tilde{Z}_{\mu}+C \tilde{Z}(t+1) .
\end{aligned}
$$

Thus, relative to simulation under $P$ with parameter uncertainty, when we simulate under $Q(\lambda), \lambda_{1}$ will have the same effect as $n^{-1 / 2} \lambda_{3}$ and $\lambda_{2}$ will have the same effect as $n^{-1 / 2} \lambda_{4}$. We can check this by comparing the values of $\lambda_{1}$ to $\lambda_{4}$ in Table 1 . Projections have been made on the basis of $n=20$ observations of $A(t+1)-A(t)$, and we can see that the ratios of $\lambda_{3}$ to $\lambda_{1}$ and $\lambda_{4}$ to $\lambda_{2}$ are both close to $(20)^{1 / 2}$ as predicted.

Now return to the results presented in Table 1 . Why are the required values of $\lambda_{1}$ to $\lambda_{4}$ positive? And why does the average risk premium per annum plotted in Figure 7 differ in the way that it does for $\lambda_{1}$ and $\lambda_{2}$ (curves A and B)?

To answer these questions, we need to analyze the impact on the mortality curve of changes in $A_{1}(t)$ and $A_{2}(t)$. Recall that we have

$$
\frac{\tilde{q}(t, x)}{\tilde{p}(t, x)}=e^{A_{1}(t)+A_{2}(t)(x+t)} .
$$

Now, if we replace $A_{1}(t)+A_{2}(t)(x+t)$ by $\bar{A}_{1}(t)+\bar{A}_{2}(t)\left(x+t-x_{0}\right)$ where $\bar{A}_{1}(t)=$ $A_{1}(t)+A_{2}(t) x_{0}$ and $\bar{A}_{2}(t)=A_{2}(t)$, then for a suitable choice of $x_{0}$ (specifically, $x_{0}=$ $\left.\hat{V}_{21} / \hat{V}_{22} \approx 62.2\right)$ the processes $\bar{A}_{1}(t)$ and $\bar{A}_{2}(t)$ become independent random walks with drift. $\bar{A}_{1}(t)$ has $Z_{1}(t)$ as its driver with market price of risk $\lambda_{1}$ and $\bar{A}_{2}(t)$ has $Z_{2}(t)$ as its driver with market price of risk $\lambda_{2}$. Under this transformation we have

$$
\begin{aligned}
\bar{A}(t+1) & =\bar{A}(t)+\bar{\mu}+\bar{C} Z(t) \\
\text { where } \bar{\mu} & =\left(\begin{array}{c}
-0.0302 \\
0.000590
\end{array}\right) \\
\text { and } \bar{C} & =\left(\begin{array}{cc}
0.01645 & 0 \\
0 & 0.001229
\end{array}\right) .
\end{aligned}
$$

We can now see that, since both diagonal elements of $\bar{C}$ are positive, a positive shock $Z_{1}(t)$ will produce a level shift in $\tilde{q}(t, x) / \tilde{p}(t, x)$ over all ages $x$ : that is, an unanticipated deterioration in longevity. A positive value of $\lambda_{1}$, in contrast, causes $\bar{A}_{1}(t)$ to be pushed downwards over time thereby enhancing improvements in longevity. So $\lambda_{1}>0$ is required to produce a positive risk premium (that is, higher expected values of $S(t)$ under $Q(\lambda)$ ).

\footnotetext{
${ }^{30}$ If we repeat cases 3 and 4, incorporating parameter uncertainty, then the remaining small differences between the cases 3 and 6 and between cases 4 and 7 essentially disappear.
} 
We have to be slightly more careful when we analyze the impact of positive shocks $Z_{2}(t)$. Specifically, for $x+t$ above age $x_{0}=62.2$, a positive value for $Z_{2}(t)$ will increase $\tilde{q}(t, x) / \tilde{p}(t, x)$ (particularly so at high ages). However, the same positive value for $Z_{2}(t)$ will cause $\tilde{q}(t, x) / \tilde{p}(t, x)$ to fall for values of $x+t$ less than 62.2. Now in our analysis we are considering a cohort who are all aged 65 at time 0 , so that $S(T)$ is constructed from the experienced mortality rates $\tilde{q}(0,65), \tilde{q}(1,65), \ldots, \tilde{q}(T-1,65)$. Since the minimum age is 65 , a positive shock in $Z_{2}(t)$ will cause an increase in each of $\tilde{q}(t-1,65), \tilde{q}(t, 65), \ldots, \tilde{q}(T-1,65)$, everything else being equal. Thus, we infer that $\lambda_{2}$ must also be positive to produce a positive risk premium.

This discussion also helps us to explain the difference between the curves corresponding to $\left(\lambda_{1}, \lambda_{2}\right)=(0.375,0)$ and $\left(\lambda_{1}, \lambda_{2}\right)=(0,0.316)$ in the lower half of Figure 7. Specifically risk adjustments to the dynamics of $A_{2}(t)$ through the use of $\lambda_{2}$ have proportionately a much greater effect on higher-age mortality than adjustments to $A_{1}(t)$ through $\lambda_{1}$. This means that the probability of survival to higher ages is much more sensitive to $\lambda_{2}$ than to $\lambda_{1}$. Thus we see that curve A in Figure 7 corresponding to $\lambda_{2}$ is flatter than curve B initially but then picks up at a much faster rate, ending up at a higher level.

\section{The Risk Premium on New Issues}

The announcement in 2004 of the 25-year EIB longevity bond will, we hope, be followed by other issues with different maturity dates and which will follow different cohorts.

Recall that the 25-year bond following the age-65 cohort (we will refer to this as the ( $T=25, x=65$ ) bond), had a 20 basis-point risk premium per annum. The question now is: What risk premiums are appropriate for bonds with different terms to maturity or that follow older or younger cohorts? It is important to address this question to ensure that possible future bonds are priced in a consistent fashion.

This question can be answered relatively easily. The key is that the market prices of risk $\lambda_{1}$ and $\lambda_{2}$ used in pricing the $(T, x)$ bond must be the same as those used in pricing the $(25,65)$ bond. Thus for each $(T, x)$ we calculate the price of the bond by determining expectations under $Q(\lambda)$ and then discounting at EIB rates as before. We then calculate the price of the bond using expectations under $P$, but then discounting at EIB rates minus the risk premium $\delta$ as in Equation (7). We then need to find the value of $\delta$ that equates the two prices under $P$ and $Q(\lambda){ }^{31}$

Recall that the only longevity bond so far proposed does not allow us to determine $\lambda$ uniquely. Instead, for any other proposed bond $(T, x)$, the risk premium $\delta(T, x, \lambda)$ will depend on $\lambda$.

Risk premia on $(T, x)$ bonds are given in Tables 2,3 , and $4 .^{32}$ We can make the following observations:

${ }^{31}$ We have not made any allowance in these calculations for parameter risk. We commented earlier that the impact of this is minimal for the $(25,65)$ bond priced with a 20 basis-point risk premium.

32 We concentrate on 20-, 25- and 30-year bonds, but, for completeness we have included infinitematurity longevity bonds (which Blake and Burrows, 2001, called survivor bonds). However, 


\section{TABLE 2}

Longevity Bond Risk Premium in Basis Points per Annum as a Function of Term to Maturity and Initial Age of Cohort. Market Price of Longevity Risk Assumed to $\mathrm{Be} \lambda=(0.375,0)$.

\begin{tabular}{lccc}
\hline & \multicolumn{3}{c}{ Initial Age of Cohort, $x$} \\
\cline { 2 - 4 } Bond Maturity $T$ & 60 & 65 & 70 \\
\hline 20 & 8.9 & 14.7 & 23.1 \\
25 & 12.7 & $\mathbf{2 0 . 0}$ & 28.7 \\
30 & 16.9 & 24.3 & 31.5 \\
$\infty$ & 22.9 & 27.2 & 32.2 \\
\hline
\end{tabular}

\section{TABLE 3}

Longevity Bond Risk Premium in Basis Points per Annum as a Function of Term to Maturity and Initial Age of Cohort. Market Price of Longevity Risk Assumed to $B e \lambda=(0,0.316)$.

\begin{tabular}{lccc}
\hline & \multicolumn{3}{c}{ Initial Age of Cohort, $x$} \\
\cline { 2 - 4 } Bond Maturity $T$ & 60 & 65 & 70 \\
\hline 20 & 4.8 & 12.4 & 26.1 \\
25 & 9.2 & $\mathbf{2 0 . 0}$ & 36.1 \\
30 & 15.0 & 27.6 & 42.3 \\
$\infty$ & 27.1 & 34.8 & 44.7 \\
\hline
\end{tabular}

\section{TABLE 4}

Longevity Bond Risk Premium in Basis Points per Annum as a Function of Term to Maturity and Initial Age of Cohort. Market Price of Longevity Risk Assumed to $B e \lambda=(0.175,0.175)$.

\begin{tabular}{lccc}
\hline & \multicolumn{3}{c}{ Initial Age of Cohort, $x$} \\
\cline { 2 - 4 } Bond Maturity $T$ & 60 & 65 & 70 \\
\hline 20 & 6.8 & 13.4 & 25.1 \\
25 & 11.0 & 20.0 & 33.3 \\
30 & 16.2 & 26.6 & 37.9 \\
$\infty$ & 25.5 & 33.7 & 39.6 \\
\hline
\end{tabular}

- In each table we see that older cohorts attract a higher risk premium. As we take younger and younger cohorts, the mortality rates get closer to zero, so even if we introduce a market price of risk, the probability of survival will still be close to 1 . In contrast, at higher ages the market price of longevity risk will have a proportionally

we note the practical difficulties associated with such bonds in dealing with the small numbers of survivors at very high ages as well as lack of reliability in mortality statistics at these ages. 


\section{FIGURE 8}

Plot of the Variances of $\log S(t)$ for the Age 60 (Solid Line), 65 (Dashed Line), and 70 (Dotted Line) Cohorts, Based on Data from 1982 to 2002, with No Allowance for Parameter Uncertainty

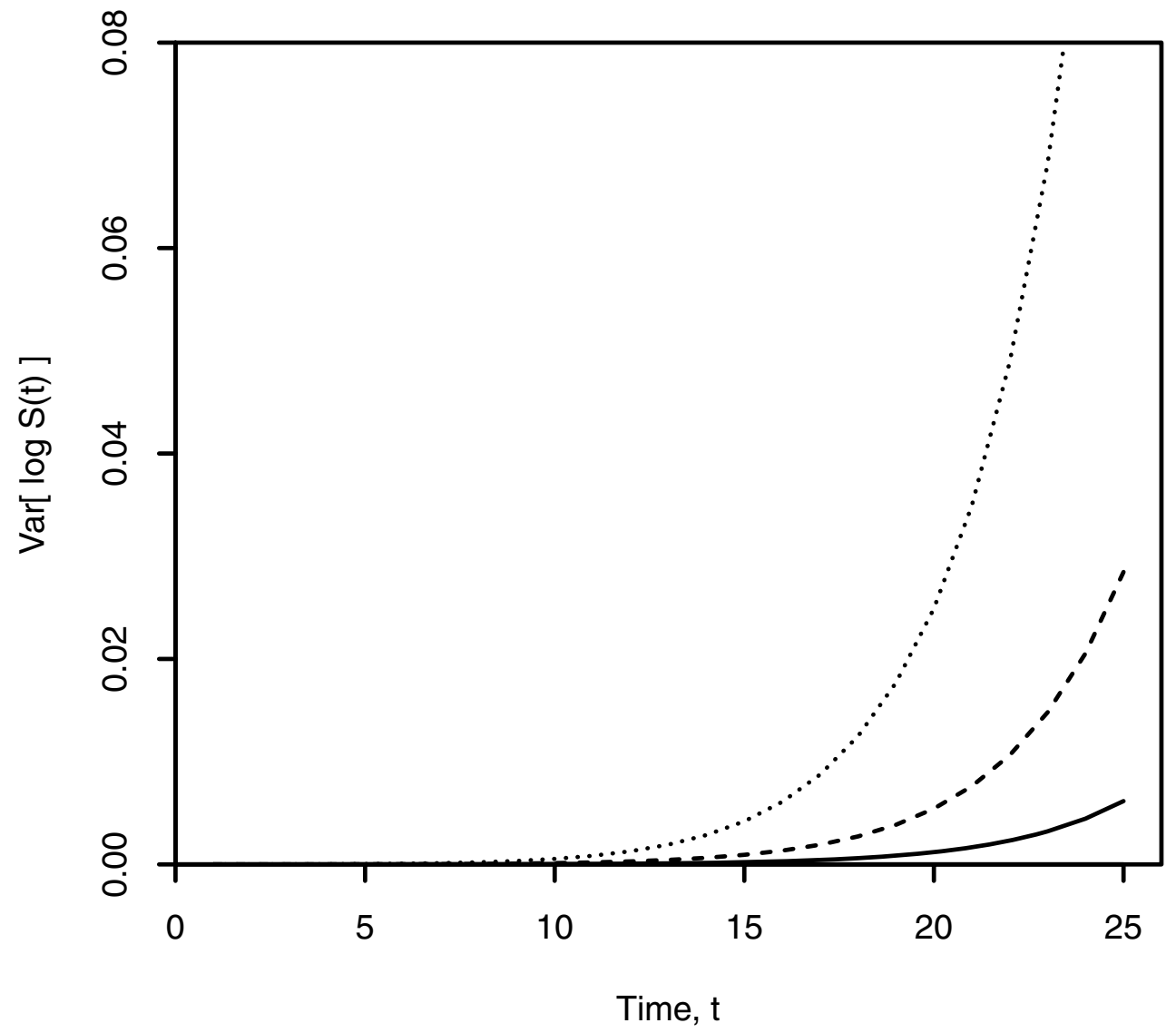

greater impact on the survival probability. These differences between ages 60, 65, and 70 are illustrated by the $\operatorname{Var}[\log S(t)]$ plot in Figure 8 . We can see that the longevity risk for the age- 60 cohort is much lower than the age-65 and age-70 cohorts. Consequently, a lower risk premium is appropriate.

- In each table, we see that the longer the maturity of the bond, the greater the risk premium. This reflects our earlier observations (for example, Figure 7, bottom) that longer-dated cashflows have a higher risk premium per annum.

- In each table, consider the diagonal running from cohort 60, term 30 up to cohort 70 , term 20. In each case the terminal age is 90 . As we move up the diagonal, there are two conflicting trends influencing the risk premium. The shortening maturity serves to push the risk premium down, ${ }^{33}$ while the increasing initial age serves to push the risk premium up. However, we can see from the table that the latter

\footnotetext{
${ }^{33}$ See Table 5 for this trend.
} 


\section{TABLE 5}

Effect of the Change of Measure from $P$ to $Q(\lambda)$ for $\lambda=(0.175$, $0.175)$ on Expected Future, Truncated Lifetimes: $e(x, T)=$ $\int_{0}^{T} E[S(t)] d t$. Upper Part of Table Shows e $(x, T)$ Under the Real-World Measure $P$ and the Lower Part of the Table Shows the Increase in $e(x, T)$ When We Change to the Risk-Neutral Measure $Q(\lambda)$.

\begin{tabular}{|c|c|c|c|c|}
\hline & & \multicolumn{3}{|c|}{ Initial Age of Cohort, $x$} \\
\hline & & 60 & 65 & 70 \\
\hline & & \multicolumn{3}{|c|}{$e_{P}(x, T)$} \\
\hline Maximum & 20 & 16.95 & 15.15 & 12.74 \\
\hline Years & 25 & 19.59 & 16.78 & 13.45 \\
\hline \multirow[t]{3}{*}{$T$} & 30 & 21.30 & 17.53 & 13.64 \\
\hline & $\infty$ & 22.43 & 17.79 & 13.66 \\
\hline & & \multicolumn{3}{|c|}{$e_{Q(\lambda)}(x, T)-e_{P}(x, T)$} \\
\hline Maximum & 20 & 0.12 & 0.20 & 0.28 \\
\hline Years & 25 & 0.28 & 0.40 & 0.47 \\
\hline \multirow[t]{2}{*}{$T$} & 30 & 0.54 & 0.65 & 0.60 \\
\hline & $\infty$ & 1.22 & 1.02 & 0.66 \\
\hline
\end{tabular}

trend dominates and the risk premium increases as we move up the diagonal. Compare, for example, one cohort currently aged 60 with another currently aged 70 and consider the contracted cashflow at age 90 . This cashflow is clearly subject to greater uncertainty for the age- 60 cohort. However, the observation above indicates that the overall impact of this greater uncertainty on the 30-year longevity bond is much reduced by the effect of discounting.

- The risk premium $\delta(T, x, \lambda)$ varies most with $(T, x)$ when $\lambda=(0,0.315)$ (Table 3$)$. The greater variation with $T$ reflects the development of the risk premium illustrated in Figure 7, bottom. The greater variation with $x$ reflects the fact that $Z_{2}(t)$ affects mortality rates in different ways at different ages. The market price of risk $\lambda_{2}$ has a positive effect on mortality at higher ages and a negative effect at lower ages.

- From Table 4 with the intermediate $\lambda=(0.175,0.175)$ we see that the risk premia lie between those given in Tables 2 and 3 .

Table 5 shows the impact in the truncated expected future lifetime $e(x, T)$ when we move from the real-world measure, $P$, to the risk-neutral measure $Q(\lambda)$ when $\lambda=$ $(0.175,0.175){ }^{34}$ The trends in this table match those in Table 4 with the exception of the trend along the diagonal from $(x, T)=(60,30)$ to $(70,20)$ where the trend is reversed. As we move upwards along the diagonal we have the same two factors working in opposite directions as before: decreasing term and increasing age. In Tables $2-4$, the impact of discounting was sufficient to allow the increasing-age effect to dominate. In Table 5 the absence of discounting means that the decreasing-term effect is dominant.

\footnotetext{
${ }^{34}$ If $\tau_{x}$ is the random future lifetime of an individual aged $x$, then $e(x, T)=E\left[\min \left\{\tau_{x}, T\right\}\right]=$ $\int_{0}^{T} E[S(t)] d t$.
} 


\section{Sensitivity to the EIB Interest Rate}

We can also investigate the impact of a change in interest rates. Specifically, let us take $\lambda$ as given but change the EIB interest rate from $4 \%$ to $5 \%$ per annum. In this case we find that the impact on the risk premium is relatively small. Specifically, if $\lambda=(0.375,0)$ then $\delta(25,65)=19.1$ basis points and if $\lambda=(0,0.315)$ then $\delta(25,65)=$ 18.9 basis points. This reduction in the risk premium reflects the relative lowering, in present-value terms, of the later, more-uncertain cashflows under the bond.

\section{The Sign of the Market Price of Risk}

In previous sections, we focused on the EIB/BNP longevity bond and used the information contained in the offer price to make inferences about the market price of risk. We concluded that this particular bond had a negative risk premium: that is, holders of the bond were being asked to pay a premium in order to reduce their exposure to longevity risk. ${ }^{35}$

Now consider a bond that allows life insurers to hedge their exposure to short-term catastrophic mortality risk in their term-insurance portfolios (for example, the Swiss Re mortality bond issued in $2003^{36}$ ). One might argue that life insurers will be prepared to pay a premium to reduce their exposure to the risk of high mortality rates. Indeed this is the case with the Swiss Re mortality bond (see, for example, Beelders and Colarossi, 2004). The problem is that this suggests that the market prices of risk in our model should take the opposite sign to those estimated in the section titled "Example: the EIB/BNP Longevity Bond."

We can offer some partial answers to this apparent paradox.

- The apparent differences between implied market prices of risk would suggest the existence of arbitrage opportunities. However, market frictions limit the ability of annuity providers and life insurers to take advantage of these opportunities. These firms can arbitrage away some differences between the different market prices of risk, for example, by exploiting natural hedging. However, there are limits to how much arbitrage they can realistically carry out because the dynamic strategies involved are not costless to implement. There might also be regulatory constraints that prevent annuity providers and life insurers from taking advantage of apparent arbitrage opportunities. Thus, as in any other "imperfect" market, a certain amount of price differentiation will remain, and we cannot rule out the possibility that the different market prices of risk might have different signs.

${ }^{35}$ Recall that the market price of risk, $\lambda$, is a displacement term that is applied to the standard normal random variable $Z(t)$. $\lambda$ can be interpreted as the instantaneous expected rate of return per unit of risk in excess of the risk-free rate of interest. For a traded asset such as a zero-coupon longevity bond, the quantity of risk is the volatility of the bond price and the instantaneous risk premium is the volatility multiplied by the market price of risk. In the previous sections we quoted average risk premiums (for example, $\delta$ for the longevity bond) over the lifetime of the asset.

${ }^{36}$ The Swiss Re bond was a three-year floating-rate bond. The bond's capital was at risk if aggregate mortality during one of the three years exceeded some high threshold. The purpose of this was to allow Swiss Re to reduce its exposure to catastrophic mortality events such as an influenza pandemic. 
- The individual lives associated with annuity and term-assurance portfolios are not subject to exactly the same rates of mortality. The age distributions of the two populations and the average terms of the policies are quite different, and it is evident from historical data that mortality improvements at different ages are not perfectly correlated. The two groups are subject to quite different levels of underwriting. It is also likely that their social backgrounds and family status are different. All of these differences will have an impact on their mortality prospects. To some degree, therefore, it might be possible to apply different market prices of risk to the different policy groups and over different age ranges.

- Annuity providers seem to be focused on the risk associated with the long-term trend in mortality (in our case, driven by a two-dimensional Brownian motion, $Z(t)$ ). In contrast, life insurers and reinsurers seem to be more focused on short-term catastrophic mortality risk (as is the case in the Swiss Re bond): it is this risk factor, much more than longevity, that is the critical determinant of a life insurer's profit or loss. In order to model this type of risk, it would be appropriate to add to our model an additional source of risk that captures more reasonably these extreme mortality risks (see, for example, Beelders and Colarossi, 2004). This additional risk will have its own market price of risk. If we combine life insurers and annuity providers, it seems quite plausible that there is, in aggregate, a significant, positive net exposure to both short-term catastrophic mortality risk and longevity risk. However, natural hedging (Cox and Lin, 2004) can only succeed at the global level (encompassing life insurers and annuity providers) if this aggregate exposure is close to zero. If these net exposures are, in reality, positive, there remains an opportunity for the financial markets to charge both life insurers and annuity providers a premium to hedge their risks.

\section{Alternative Models}

\section{General Models}

We have deliberately chosen to use a simple (linear) parametric form for $\log \tilde{q}(t, x) / \tilde{p}(t, x)$. In part this is because the data seem to justify this assumption over the 60-90 age range (see, for example, Figure 1). In addition, the simple model allows us to focus attention on the key issues in this article: highlighting the risk associated with future mortality-linked cashflows; and the calculation of the risk-adjusted price of these cashflows.

A variety of alternative stochastic models have, of course, been proposed (see, for example, Cairns, Blake, and Dowd, 2006, and references therein). However, rigorous statistical analysis has, in the main, been limited to the the approach proposed by Lee and Carter (1992) and their successors (see, for example, Brouhns, Denuit, and Vermunt, 2002; and Renshaw and Haberman, 2003). The model analyzed in this article might be considered as a special case of a two-factor Lee and Carter model (Renshaw and Haberman, 2003) ${ }^{37}$.

Despite the relative simplicity of our model we have chosen to use two stochastic factors rather than one. We did so partly because our earlier analysis suggests that

\footnotetext{
${ }^{37}$ Here, though, we model $\log \tilde{q}(t, x) / \tilde{p}(t, x)$ in place of the usual $\log \tilde{m}(t, x)$ where $\tilde{m}(t, x)$ is the central death rate.
} 
we need two factors to get the best fit. However, we also did so because our later analysis highlights the importance of the longer-term longevity risks (that is, the risks associated with survivorship to very old ages), and we need the second mortality factor to model these particular risks adequately. Finally, we wish to consider a range of bonds with different maturity dates and following different cohorts, and this merits an additional factor if the historical data supports it.

\section{Modelling the Cohort Effect}

A number of authors have recently focused attention on what has become known as the cohort effect. These analyses (see, for example, Willets, 1999, 2004; Richards, Kirkby, and Currie, 2005; and MacMinn et al., 2005) have demonstrated that, for a fixed age $x$, the improvement in mortality from one calendar year to the next is critically dependent on the year of birth (that is $t-x$ ). Richards, Kirkby, and Currie found, for example, that the largest improvements in mortality rates in England and Wales have been consistently experienced by individuals born around 1930. We have not attempted to capture this effect in the current article. A recent paper by Renshaw and Haberman (2006) has adapted the Lee and Carter approach to incorporate a cohort effect and we anticipate more work along these lines in the coming years.

We can note that the change in pattern in $A_{1}(t)$ and $A_{2}(t)$ around 1985, observable in Figure 2, is also consistent with the cohort effect. ${ }^{38}$ For example, suppose the slope parameter, $A_{2}(t)$, would naturally be constant in the absence of a cohort effect. If we then introduce a cohort effect, we would find that as the "golden" cohort moves through the 60-90 age range, we would initially see the fitted curve steepen for 15 years and then fall back to its original slope over the next 15 years (that is, $A_{2}(t)$ would rise from its normal stable level and then fall back). In Figure 2, we can see that $A_{2}(t)$ was reasonably level, before starting to climb after 1985. If the cohort effect persists then we might anticipate that $A_{2}(t)$ will start falling around the time when the 1930 cohort passes age 75 (the mid-point of our age 60-90 data sets). Thus, we might expect to see $A_{2}(t)$ start to fall back again over the next few years, instead of continuing to rise.

\section{CONCLUSIONS}

In this article, we have used a simple two-factor model for the development of the mortality curve over time that seems, nevertheless, to fit the data well. The model allows us to simulate the distribution of a survivor index over various time horizons under both the real-world probability measure and under a variety of possible risk-adjusted measures. By taking expectations under the latter measure, this model enables us to price the longevity risk inherent in longevity bonds, given the known longevity risk premium (of 20 basis points) contained in the world's first longevity bond, namely the November 2004 EIB 25-year bond designed by BNP Paribas with a reference cohort of 65-year-old English and Welsh males. The chosen model is well suited to pricing longevity bonds. For other types of contracts, that involve, for example, derivative

\footnotetext{
${ }^{38}$ Note that some, but not all, simulated paths of $A(t)$ using the random walk model also exhibit apparent changes in the trend over periods of up to twenty years. That is, the random-walk model is just as consistent with the historical patterns in Figure 2 as the pattern that would be induced by the cohort effect.
} 
characteristics on future mortality rates (such as guaranteed annuity options) models formulated within the forward-mortality model (such as the Olivier-Smith model described in Olivier and Jeffrey, 2004) or the mortality-market model (Cairns, Blake, and Dowd, 2006) frameworks are likely to prove more efficient to implement.

We find that the premium increases with both term and the initial age of the reference cohort. In the latter case, this is caused by the greater volatility that is associated with the higher mortality rates of older people compared with younger people. For example, in the worst-case scenario considered (where the entire longevity risk premium is associated with the second (i.e., volatility) factor), the premium for a 30-year bond with a reference cohort aged 70 is 42.3 basis points.

Another key finding of the article is that the reference cohort's initial age is more important for determining the premium than the bond's maturity. To illustrate, again in the context of the worst-case scenario, the premium for a 20-year bond with a reference cohort aged 70 is 26.1 basis points, whereas the premium for a 30-year bond with a reference cohort aged 60 is 15.0 basis points. This shows that the greater uncertainty in death rates at higher ages dominates the greater discounting of the more distant cash flows of longer maturing bonds.

These findings suggest that open-ended survivor bonds that continue to pay out as long as members of the reference cohort are still alive would not have an excessively high longevity risk premium. However, they might be unattractive in other respects, such as the administrative inconvenience associated with paying very small coupons fifty years or so after the bond was issued. So fixed-term longevity bonds might well dominate for practical considerations. Our results also suggest that fixedterm longevity bonds might also be favored by investors wishing to avoid ultra-long longevity risk being dominated by parameter risk.

We propose in future research to investigate alternatives to the random walk model with drift used here. Possibilities include models drawn from the ARIMA class of time series models. ${ }^{39}$ By taking this approach, we will be investigating the important issue of model risk in addition to the parameter risk considered in this article.

In this article, we have assumed that the fitted values of $A(t)$ are known with certainty. A further line of research is to relax this assumption and to use instead filtering approaches or Markov Chain Monte Carlo (MCMC) methods to estimate jointly the posterior distribution of the parameters and of the current values of $A(t)$.

\section{Appendix A}

\section{THE EIB/BNP LONGEVITY BOND}

The EIB/BNP Paribas longevity bond makes reference to a cohort (aged $x$ at time 0 ) index that is calculated along the following lines. We start, for convenience, by letting $t=0$ correspond to the beginning of 2003 and set the reference index $S(0)=$ 1. Changes in the reference index, $S(t)$, from one year to the next are determined by reference to national mortality rates which are made publicly available. Thus,

\footnotetext{
${ }^{39}$ The model used here is classified as an $\operatorname{ARIMA}(0,1,0)$ model. Elsewhere (Cairns, Blake, Dawson, and Dowd, 2005) we have used an $\operatorname{ARIMA}(1,1,0)$ model with similar results to those in this article.
} 
for $t=1,2, \ldots, S(t+1)=S(t)(1-\tilde{m}(t, x))$, where $\tilde{m}(t, x)$ is the central death rate for individuals aged $x+t$ in year $t$ (that is, age 65 in 2003, age 66 in 2004, and so on) published by the UK Office for National Statistics (ONS).

The publication by the ONS of central death rates contrasts with the mortality rates $(\tilde{q}(t, x))$ published, for example, by the UK Government Actuary's Department (GAD) (for example, as with those for 2002 plotted in Figure 1). For a full discussion of the differences between $\tilde{q}(t, x)$ and $\tilde{m}(t, x)$ the reader is referred to standard texts such as Benjamin and Pollard (1993) or Bowers et al. (1986). A key approximation connecting the two is given by

$$
\tilde{m}(t, x) \approx \frac{\tilde{q}(t, x)}{1-\frac{1}{2} \tilde{q}(t, x)} .
$$

We have used this approximation in our simulations of $S(t)$.

\section{Appendix B}

\section{Simulation of the Normal-Inverse-Wishart Distribution}

Equation (5) requires simulation of $V \mid D$ using its posterior distribution, the Wishart $\left(n-1, n^{-1} \hat{V}^{-1}\right)$ distribution. It is more instructive to show how to simulate from the Wishart distribution than it is to write down its density function. Thus:

- Let $S$ be the upper triangular matrix that satisfies $S S^{\prime}=n^{-1} \hat{V}^{-1}$.

- Now simulate $n-1$ i.i.d. vectors $\alpha_{1}, \ldots, \alpha_{n-1} \sim \operatorname{MVN}\left(0, S S^{\prime}\right)$ : that is, let $\alpha_{i}=$ $S Z_{i}$, where $Z_{i}$ is a standard $n$-dimensional normal random variable (that is, the individual elements of each $Z_{i}$ are independent normal random variables with mean 0 and variance 1 ).

- Let $X=\sum_{i=1}^{n-1} \alpha_{i} \alpha_{i}^{\prime}$.

- Then $X$ has a Wishart $\left(n-1, n^{-1} \hat{V}^{-1}\right)$ distribution.

- Our final step is to invert $X$ to get our simulated covariance matrix: that is, $V=$ $X^{-1}$.

Note that $E[X]=\frac{n-1}{n} \hat{V}^{-1}$. Thus the distribution of the simulated matrices $V=X^{-1}$ will be centered close to $\hat{V}$ itself.

The second step of simulating from the Normal-Inverse-Wishart posterior distribution is to take the simulated $V$ from the steps above and then sample $\mu$ from a multivariate normal distribution with mean $\hat{\mu}$ and covariance matrix $n^{-1} V$. This can be simulated in the usual way.

\section{Appendix C ILLUSTRATION OF ACCUMULATEd VARIANCE}

Conside a random walk in which $W(0)=0$ and, for each $t, W(t+1)=W(t)+Z(t+$ $1)$, where $Z(1), Z(2), \ldots$ is a sequence of i.i.d. standard normal random variables.

The way in which $\operatorname{Var}(\log S(t))$ builds up is similar to $Y(T)=\sum_{t=1}^{T} W(t)$. Since $W(t)=$ $Z(1)+\ldots+Z(t)$ we have

$$
Y(T)=T Z(1)+(T-1) Z(2)+\ldots+2 Z(T-1)+Z(T) .
$$


This has variance $\sum_{t=1}^{T} t^{2}=\frac{1}{6} T(T+1)(2 T+1)$. In contrast $\operatorname{Var} W(T)=T$.

If we introduce a drift, $\lambda$, into the process (that is, the $Z(t)$ are i.i.d. $N(\lambda, 1)$ ) (thereby having the same effect as a market price of risk) then

$$
Y(T)=\frac{1}{2} T(T+1) \lambda+T Z(1)+(T-1) Z(2)+\ldots+2 Z(T-1)+Z(T) .
$$

This does not affect the variance if $\lambda$ is known. However, if $\lambda$ is unknown with mean $\hat{\lambda}$ and variance $\sigma^{2}$, then

$$
\operatorname{Var} Y(T)=\frac{1}{6} T(T+1)(2 T+1)+\frac{1}{4} T^{2}(T+1)^{2} \sigma^{2} .
$$

As a consequence, uncertainty in $\lambda$ causes greater uncertainty in $Y(T)$ than does the underlying volatility in $W(T)$ as $T$ gets larger (that is, the $\frac{1}{4} T^{2}(T+1)^{2} \sigma^{2}$ term dominates as $T$ gets larger).

\section{References}

Beelders, O., and D. Colarossi, 2004, Modelling Mortality Risk with Extreme Value Theory: The Case of Swiss Re's Mortality-Indexed Bonds, GARP Risk Review, 19: 26-30.

Benjamin, B., and J. H. Pollard, 1993, The Analysis of Mortality and Other Actuarial Statistics, 3rd edition (London: Institute of Actuaries).

Biffis, E., 2005, Affine Processes for Dynamic Mortality and Actuarial Valuations, Insurance: Mathematics and Economics, 37: 443-468.

Blake, D., and W. Burrows, 2001, Survivor Bonds: Helping to Hedge Mortality Risk, Journal of Risk and Insurance, 68: 339-348.

Blake, D., A. J. G. Cairns, and K. Dowd, 2006, Living with Mortality: Longevity Bonds and Other Mortality-Linked Securities, British Actuarial Journal, in press.

Bowers, N. L., H. U. Gerber, J. C. Hickman, D. A. Jones, and C. J. Nesbitt, 1986, Actuarial Mathematics (Itasca: Society of Actuaries).

Brouhns, N., M. Denuit, and J. K. Vermunt, 2002, A Poisson Log-Bilinear Regression Approach to the Construction of Projected Life Tables, Insurance: Mathematics and Economics, 31: 373-393.

Cairns, A. J. G., 2000, A Discussion of Parameter and Model Uncertainty in Insurance, Insurance: Mathematics and Economics, 27: 313-330.

Cairns, A. J. G., D. Blake, and K. Dowd, 2006, Pricing Death: Frameworks for the Valuation and Securitization of Mortality Risk, ASTIN Bulletin, 36: 79-120.

Cairns, A. J. G., D. Blake, P. Dawson, and K. Dowd, 2005, Pricing the Risk on Longevity Bonds, Life and Pensions, October: 41-44.

Cowley, A., and J. D. Cummins, 2005, Securitization of Life Insurance Assets and Liabilities, Journal of Risk and Insurance, 72: 193-226.

Cox, S., J. Fairchild, and H. Pedersen, 2000, Economic Aspects of Securitisation of Risk, ASTIN Bulletin, 30: 157-193. 
Cox, S., Y. Lin, and S. Wang, 2005, Pricing Longevity Bonds, Presentation at the 1st International Conference on Longevity Risk and Capital Market Solutions, Cass Business School, London, February, 2005.

Cox, S., and Y. Lin, 2004, Natural Hedging of Life and Annuity Mortality Risks, In the Proceedings of the 14th International AFIR Colloquium, Boston, pp. 483-507.

Currie I. D., M. Durban, and P. H. C. Eilers, 2004, Smoothing and Forecasting Mortality Rates, Statistical Modelling, 4: 279-298.

Dahl, M., 2004, Stochastic Mortality in Life Insurance: Market Reserves and Mortality-Linked Insurance Contracts, Insurance: Mathematics and Economics, 35: 113136.

Dahl, M., and T. Møller, 2005, Valuation and Hedging of Life Insurance Liabilities with Systematic Mortality Risk, In the Proceedings of the 15th International AFIR Colloquium, Zurich, Available online at <http://www.afir2005.ch>.

Denuit, M., P. Devolder, and A-C. Goderniaux, 2004, Securitization of Longevity Risk: Pricing Survival Bonds with Wang Transform in the Lee-Carter Framework, Preprint, Université Catholique de Louvain.

Dowd, K., D. Blake, A. Cairns, and P. Dawson, 2006, Survivor Swaps, Journal of Risk and Insurance, 73: 1-17.

Gelman, A., J. Carlin, H. Stern, and D. Rubin, 1995, Bayesian Data Analysis (London: Chapman and Hall).

Lane, M. N., 2000, Pricing Risk Transfer Transactions, ASTIN Bulletin, 30: 259-293.

Lee, R. D., and L. R. Carter, 1992, Modeling and Forecasting U.S. Mortality, Journal of the American Statistical Association, 87: 659-675.

Lin, Y., and S. H. Cox, 2004, Securitization of Mortality Risks in Life Annuities. Journal of Risk and Insurance, 72: 227-252.

MacMinn, R., K. Ostaszewski, R. Thiagarajah, and J. F. Weber, 2005, An Investigation of Select Birth Cohorts, In Living to 100 and Beyond (Schaumburg, IL: Society of Actuaries).

Milevsky, M. A., and S. D. Promislow, 2001, Mortality Derivatives and the Option to Annuitise, Insurance: Mathematics and Economics, 29: 299-318.

Miltersen, K. R., and S.-A. Persson, 2005, Is Mortality Dead? Stochastic Forward Force of Mortality Determined by No Arbitrage, Working paper, University of Bergen.

Olivier, P., and T. Jeffery, 2004, Stochastic Mortality Models, Presentation to the Society of Actuaries of Ireland. See <http://www.actuaries.ie/PastCalendar $>$.

Perks, W., 1932, On Some Experiments in the Graduation of Mortality Statistics, Journal of the Institute of Actuaries, 63: 12-57.

Renshaw, A. E., and S. Haberman, 2003, Lee-Carter Mortality Forecasting with Agespecific Enhancement, Insurance: Mathematics and Economics, 33: 255-272.

Renshaw, A. E., and S. Haberman, 2006, A Cohort-Based Extension to the Lee-Carter Model for Mortality Reduction Factors, Insurance: Mathematics and Economics, 38: 556-570.

Richards, S. J., J. G. Kirkby, and I. D. Currie, 2005, The Importance of Year of Birth in Two-dimensional Mortality Data, British Actuarial Journal. 
Schrager, D. F., 2006, Affine Stochastic Mortality. Insurance: Mathematics and Economics, 38: 81-97.

Wang, S. S., 2000, A Class of Distortion Operations for Pricing Financial and Insurance Risks, Journal of Risk and Insurance, 67: 15-36.

Wang, S. S., 2002, A Universal Framework for Pricing Financial and Insurance Risks, ASTIN Bulletin, 32: 213-234.

Wang, S. S., 2003, Equilibrium Pricing Transforms: New Results Using Buhlmann's 1980 Economic Model, ASTIN Bulletin, 33: 57-73.

Willets, R. C., 1999, Mortality in the Next Millennium, Paper presented to the Staple Inn Actuarial Society. See $<$ http://www.slas.org.uk $>$

Willets, R. C., 2004, The Cohort Effect: Insights and Explanations, British Actuarial Journal, 10: 833-877. 